\title{
Médiévales
}

Langues, Textes, Histoire

48 | printemps 2005

Princes et princesses à la fin du Moyen Âge

\section{Isabelle de France, sœur de saint Louis : la vierge savante}

Une étude de la Vie d'Isabelle de France écrite par Agnès d'Harcourt

Isabelle de France, Saint Louis' Sister : the Literate Virgin.

\section{Anne-Hélène Allirot}

\section{OpenEdition}

\section{Journals}

Édition électronique

URL : https://journals.openedition.org/medievales/1050

DOI : 10.4000/medievales. 1050

ISSN : 1777-5892

\section{Éditeur}

Presses universitaires de Vincennes

Édition imprimée

Date de publication : 1 juin 2005

Pagination : $55-98$

ISBN : 2-84292-169-0

ISSN : 0751-2708

\section{Référence électronique}

Anne-Hélène Allirot, «Isabelle de France, sœur de saint Louis : la vierge savante », Médiévales [En ligne], 48 I printemps 2005, mis en ligne le 02 mars 2007, consulté le 22 avril 2022. URL : http:// journals.openedition.org/medievales/1050 ; DOI : https://doi.org/10.4000/medievales.1050

Ce document a été généré automatiquement le 22 avril 2022.

Tous droits réservés 


\title{
Isabelle de France, sœur de saint Louis : la vierge savante
}

\author{
Une étude de la Vie d'Isabelle de France écrite par Agnès d'Harcourt \\ Isabelle de France, Saint Louis' Sister : the Literate Virgin.
}

\author{
Anne-Hélène Allirot
}

1 Isabelle de France, unique sœur de Louis IX, est née en mars 1225. Elle fait vœu de chasteté et refuse tous les mariages qu'on lui propose. Elle fonde le monastère des Clarisses de Longchamp en 1255 et s'y retire. Elle meurt le 23 février 1270. Les biographes de saint Louis sont peu prolixes à son sujet: Guillaume de Saint-Pathus signale qu'elle reçut, comme ses frères, l'éducation religieuse de Blanche de Castille ${ }^{1}$. Jean de Joinville la mentionne brièvement en tant que fondatrice de l'abbaye de Longchamp ${ }^{2}$. Ce n'est qu'à travers la Vie d'Isabelle de France ${ }^{3}$, récit hagiographique rédigé par son ancienne dame de compagnie, Agnès d'Harcourt, à la demande de Charles d'Anjou, que l'on découvre en elle un personnage essentiel de l'entourage de saint Louis. Il s'agit de l'un des premiers textes en prose et en français dont l'auteur est une femme, de plus clairement identifiée. Malgré son extrême richesse, ce texte n'a suscité que peu d'analyses historiques ${ }^{4}$.

2 Agnès d'Harcourt appartient à une grande famille de lettrés. Elle est la fille de Jean I ${ }^{\text {er }}$ d'Harcourt, seigneur normand, compagnon et ami de saint Louis, et de sa troisième femme Blanche d'Avaugour. Ses oncles Raoul et Robert, maîtres en décret, chanoines et conseillers de Philippe Le Bel, ont fondé en 1280 le collège d'Harcourt à Paris ${ }^{5}$. Dame de compagnie d'Isabelle de France, elle entre aux Clarisses de Longchamp en 1260, et y occupe la charge d'abbesse de 1263 à 1279 et de 1281 à 1286 . Elle meurt en 1289. Sa sœur Jeanne d'Harcourt occupe à son tour la même charge de 1294 à $1298^{6}$.

3 La date de rédaction du texte est nécessairement comprise entre 1270, date de la mort d'Isabelle, et 1289, date de la mort d'Agnès. La commande de Charles d'Anjou, frère de la princesse, ne peut avoir lieu qu'avant $1285^{7}$. Agnès d'Harcourt précise lors d'un récit de miracle : «Je, sœur Agnes de Harcourt, qui adonc estois en l'office d'abbesse $»^{8}$, sousentendant qu'elle ne l'est plus lorsqu'elle écrit. Son office s'interrompt effectivement 
entre 1279 et 1281 : le texte est donc peut-être rédigé dans cet intervalle, dans le contexte de l'enquête en vue de la canonisation de Louis IX. Le roi meurt en 1270, peu après sa sœur : le pape Grégoire X lance une première enquête à son sujet en 1272. Mais Nicolas III réclame en 1278 une documentation plus approfondie et rigoureuse. La demande de Charles d'Anjou à Agnès d'Harcourt intervient dans cette période, pour appuyer l'idée d'une sainteté dynastique capétienne. Le procès et les auditions de témoins durent de mai 1282 à mars 1283. Mais le roi n'est canonisé que le 4 août $1297^{9}$.

4 Le texte relate la vie d'Isabelle et ses miracles. À travers ce récit, l'auteur tente de démontrer la sainteté d'Isabelle, laïque et membre de la famille royale, dans l'optique de son éventuelle canonisation. Quels sont les traits originaux qui ressortent de cette démonstration ? L'auteur cherche-t-elle à présenter Isabelle comme un double de Claire d'Assise ou plutôt comme un double féminin de son frère, le roi Louis IX ?

Un plaidoyer pour la canonisation d'Isabelle

5 La rédaction de la Vie d'Isabelle vise à obtenir le déclenchement d'une enquête de canonisation comme celle en cours pour son frère Louis IX. Charles d'Anjou a demandé que l'une des sœurs du monastère rédige la Vie, et l'abbesse a accepté cette tâche. Le roi de Sicile semble particulièrement attaché au caractère héréditaire et familial des vertus de son frère : en 1270, il tente de garder les reliques de Louis IX dans son royaume de Sicile. Il témoigne en 1282 au procès de canonisation, en deuxième position après Philippe III. Dans sa déposition, il décrit la bonne éducation maternelle de Louis IX, mais aussi de ses frères Robert comte d'Artois et Alphonse, ainsi que de sa sœur Isabelle ${ }^{10}$. La demande de Charles d'Anjou s'inscrit donc dans une entreprise délibérée de glorification de la lignée capétienne. Piété profonde et propagande sont sans doute liées au cœur de ce que l'on peut appeler une "entreprise de famille ${ }^{11}$. Le prestige qu'apporterait la canonisation de Louis IX serait ainsi renforcé par celle d'Isabelle.

6 De manière classique, Agnès d'Harcourt insiste sur la noblesse de la lignée dont Isabelle est extraite. Mais cette présentation s'inscrit ici dans le cadre exceptionnel de la famille royale de France: "Notre saincte mere et dame madame Isabeau fust extraicte de royale lignee $\aleph^{12}$. Les membres de cette famille tiennent une grande place dans la narration comme témoins et acteurs de la vie d'Isabelle, en particulier sa mère Blanche de Castille, son frère Louis IX et sa belle-sœur Marguerite de Provence. Le prologue présente les parents d'Isabelle, «fille de tres noble roy Louis de France [...] et [...] fille de la tres noble reine de France, madame la reine Blanche ${ }^{13}$. Le père d'Isabelle, Louis VIII, est lui-même « fils du roy Philippes », Philippe Auguste. La lignée maternelle de la princesse est également mise en valeur: Blanche de Castille est «fille du roy d'Espagne ${ }^{14}$ ", Alphonse VIII roi de Castille.

7 Les titulatures des filles du roi de France manifestent de façon plus visible l'importance du lignage royal autour de 1250 : on est dite «fille du roi » du vivant de ce dernier, " fille de roi " si celui-ci est mort. Ce titre attribué ici à Isabelle doit cependant être considéré avec précaution, le texte ayant été légèrement remanié au XVII ${ }^{\mathrm{e}}$ siècle. D'autres exemples existent néanmoins pour les descendantes de saint Louis dans la même période : après son mariage en 1255, Isabelle, fille de Louis IX, est appelée " fille du roi de France, par la grâce de Dieu reine de Navarre et comtesse palatine de Champagne et de Brie » : son mariage n'efface pas l'ascendance royale ${ }^{15}$.

8 L'auteur rappelle la force des liens affectifs qui unissent la princesse, seule fille restée en vie, à ses parents : «Le pere et la mere n'avoient plus de filles, et merveilleusement l'aimoient $\aleph^{16}$. Cet amour parental, très présent, souligne la force du lien de parenté. 
Isabelle apparait comme la descendante d'une lignée illustre, et comme membre d'une famille royale soudée par l'amour et unie par les liens du sang. La famille a participé à la naissance de sa vocation, et elle contribue à la reconnaissance de son parcours d'exception. Le prestige d'Isabelle doit être partagé avec la famille royale, tout comme la princesse bénéficie des qualités héréditaires de la lignée. La sainteté est présentée ainsi comme dynastique.

9 Agnès d'Harcourt emploie une démarche hagiographique assez classique. Elle ne donne aucune date, mais il est possible de situer chronologiquement les évènements relatés : l'enfance et l'adolescence d'Isabelle entre 1225 et 1254, la fondation du monastère des Clarisses de Longchamp entre 1254 et 1260, puis la vie monastique et le trépas d'Isabelle dans les années 1260-1270. Enfin, les miracles posthumes cités ont pu se dérouler entre 1270 et 1281 au plus tard. Isabelle est dite " nostre saincte et benoiste dame et mere $»^{17}$ : elle est placée dans un double rapport, dominant et maternel, envers les religieuses de l'abbaye. La princesse est également " mirouër d'innocence, exemplaire de penitence » et " escolle de toutes bonnes mœurs " ${ }^{18}$. Isabelle, par sa " vraye noblesse », qui est celle de l'âme et non celle du sang, est un exemple donné à lire aux autres princesses et aux religieuses de Longchamp ${ }^{19}$.

10 L'œuvre dépasse cependant ce cadre classique par l'engagement personnel de l'auteur sur la véracité de son récit: Agnès d'Harcourt veut faire œuvre d'historienne. Elle rapporte des faits vus ou entendus par elle-même ou par des témoins directs, et prend toujours soin de citer la source et les circonstances du témoignage : «Je, sœur Agnes de Harecourt, oÿ ceste chose de la bouche monseigneur le roy saint Louys qui nous le raconta $»^{20}$. Elle précise quand elle le peut le plus grand nombre possible de témoins dignes de confiance. L'exploitation de deux lettres pontificales - "Nous en avons encores les lettres en nostre abbaye $»^{21}$ - montre, chez l'auteur, une forte conscience de l'importance historique de la preuve écrite ${ }^{22}$.

11 De quelles sources Agnès d'Harcourt a-t-elle pu s'inspirer, tant sur la forme que sur le fond? Tout d'abord, les textes concernant sainte Claire d'Assise et sainte Élisabeth de Hongrie ont pu lui fournir un modèle. De nombreux traits de la vie de Claire se retrouvent dans celle d'Isabelle ${ }^{23}$. Élisabeth de Hongrie est, comme Isabelle, une princesse de sang royal dont la piété se manifeste dès le plus jeune âge ${ }^{24}$. Son hagiographie la plus ancienne, le Libellus quattuor ancillarum, est fondé sur les souvenirs de ses quatre dames de compagnie ou domestiques ${ }^{25}$. L'une d'elles, Isentrude, est une ancilla, mais aussi une nobilis femina très proche d'élisabeth ${ }^{26}$. Blanche de Castille avait une dévotion particulière pour la princesse sainte ${ }^{27}$ : peut-être le texte était-il connu à la cour royale. La Vie d'Isabelle est, de la même manière, le témoignage d'une ancilla et nobilis femina, confidente de la sainte.

12 Agnès d'Harcourt a pu également lire les ouvrages de Geoffroy de Beaulieu et Guillaume de Chartres sur Louis IX. La Vita de Geoffroy de Beaulieu ${ }^{28}$, rédigée en 1272-1273 en vue du procès de canonisation du roi, présente de nombreux thèmes similaires à ceux de la Vie d'Isabelle: enfance et éducation, humilité des mœurs, charité aux pauvres, pénitence, constructions pieuses. Guillaume de Chartres, qui écrit entre 1275 et 1282, complète cette Vita et lui adjoint une liste de miracles posthumes du roi ${ }^{29}$. Comme ces biographes de Louis IX, Agnès d'Harcourt propose des matériaux pour une enquête sur la sainteté d'Isabelle; elle recueille le plus grand nombre de témoignages possibles, et les plus dignes de foi. La Vie d'Isabelle se présente comme une enquête officieuse en préliminaire à un futur procès de canonisation. 
13 La sainteté d'Isabelle est une affaire de famille, mais également une affaire de femmes. Dans le texte, ces dernières dominent en nombre: trente-sept femmes sont mentionnées par Agnès d'Harcourt pour vingt-huit hommes. Il s'agit d'abord de l'entourage d'Isabelle à la cour, sa mère, ses belles-sœurs, ses dames de compagnie ; puis le monastère des Clarisses de Longchamp devient le théâtre des événements, monde clos et, à quelques exceptions près, entièrement féminin. Les témoins des miracles sont les premières sœurs du couvent, pratiquement toutes nobles. Elles sont citées dans le manuscrit français 11662 de la Bibliothèque nationale, qui contient la liste des sœurs de l'abbaye de Longchamp. Agnès d'Anéri est la première abbesse du monastère, de 1260 à 1262. Julienne de Troyes est abbesse après Agnès d'Harcourt en 1279. Ade ou Ode de Reims est une des premières nonnes venues au couvent. Alix de Mucedent est une nonne qui a atteint l'âge de cent un ans ${ }^{30}$. Agnès d'Harcourt a choisi ses témoins parmi les femmes les plus remarquables du couvent.

14 L'auteur laisse souvent la parole aux personnages féminins et les cite au style direct. On «entend» ainsi parler Isabelle: «Je propose qu'il soit donné a Nostre Seigneur, car c'est le premier que je filasse oncques $»^{31}$, mais également certaines Clarisses de Longchamp, comme Marie de Tremblay. Agnès d'Harcourt elle-même prend la parole : « Dame, dictes moy pour Dieu, si vous plaist, pourquoy vous avez mis ce nom en nostre abbaye $\|^{32}$. Les voix féminines participent à la construction du récit et le structurent davantage que les voix masculines. Or, il est rare que la parole des femmes soit ainsi jugée digne de mémoire dans les sources médiévales, alors qu'il leur est notamment interdit de prêcher ${ }^{33}$.

15 Le plus souvent, les vies de saintes sont écrites par leur confesseur. Eudes de Rosny est celui d'Isabelle. C'est un théologien franciscain, qui a sans doute étudié avec Alexandre de Halès et succédé comme maître à Guibert de Tournai en 1260. Il est encore actif en 1272, comme en témoignent des sermons. Il est peut-être décédé au moment où Charles d'Anjou désire faire rédiger la Vie de sa sœur ${ }^{34}$. Cela expliquerait que ce dernier se soit tourné vers l'ancienne dame de compagnie de la princesse. Dans le texte d'Agnès d'Harcourt, Eudes de Rosny cherche à tempérer Isabelle : « Dame, il faut bien que vous partissiez et que vous vous esbatissiez, il ne depleust pas a Nostre Seigneur si vous prissiez un peu de recreation $»^{35}$. Ce topos des vies de saints permet de mettre en valeur la dévotion d'Isabelle. C'est aussi un moyen pour l'abbesse de Longchamp de se donner comme témoin privilégié de l'affirmation d'une sainte laïque face à son confesseur ${ }^{36}$. Mais Agnès d'Harcourt insiste également sur l'extrême respect de la princesse à l'égard de Eudes de Rosny ${ }^{37}$. Le récit de l'abbesse n'échappe pas au contrôle masculin: la demande émane de Charles d'Anjou et doit convenir pour une lecture par des clercs en vue d'une éventuelle canonisation.

Isabelle, la vierge savante : une sainte dans la famille royale

16 La démonstration de la sainteté d'Isabelle utilise évidemment de nombreux stéréotypes hagiographiques. L'auteur démontre d'abord que sa vocation est précoce et aussitôt reconnue comme telle de façon unanime. Des signes physiques se manifestent en premier lieu : sa beauté corporelle est le reflet de sa beauté morale. Par la suite, c'est l'ensemble du comportement de l'adolescente qui signale sa sainteté : elle ne partage pas les jeux des autres jeunes filles et préfère réaliser divers travaux de couture pour l'Église. Elle refuse le luxe de la cour ou les vêtements offerts par sa mère. La prière est sa principale activité. Il est fréquent que l'enfance du saint soit ainsi marquée par le 
rejet de la famille terrestre ${ }^{38}$. Mais ce rejet est particulièrement marquant dans le cas d'Isabelle, qui évolue dans le cadre de la cour royale.

L'intervention du surnaturel est un autre critère nécessaire à toute saintetée ${ }^{39}$. Certains indices liés au trépas d'Isabelle sont des lieux communs utilisés depuis le haut Moyen Âge, comme le chant des anges que l'on peut entendre la nuit de sa mort ${ }^{40}$. Sorti de son cercueil au bout de neuf jours, le corps d'Isabelle n'est nullement corrompu, ses membres sont beaux «comme d'un tendre enfant $»^{41}$. Les dépouilles des saints, incorruptibles, ne peuvent connaître le même sort que celles du commun des mortels. Le corps devient tendre comme une chair d'enfant: c'est le signe de l'élection, notamment dans le récit de la mort de François d'Assise par Thomas de Celano ${ }^{42}$. L'odeur agréable qui se dégage du corps est le parfum des saints ${ }^{43}$. Les sœurs prennent alors la robe de la princesse comme relique, et le public cherche à toucher le corps avec des objets qui deviennent à leur tour des reliques ${ }^{44}$.

Quarante miracles sont relatés dans la Vie d'Isabelle, dont quatre survenus avant son trépas. La présence de miracles in vita est exceptionnelle pour la période : en effet, les papes ont imposé une définition rigoureuse de la perfection chrétienne dans la première moitié du XIII ${ }^{e}$ siècle, notamment Innocent III (1198-1216) et Grégoire IX (1227-1241). Les miracles demeurent indispensables à la procédure de canonisation, mais ils ne doivent servir qu'à manifester après la mort du saint ses mérites éminents et l'excellence de sa vie ${ }^{45}$. La Vie d'Isabelle reproduit ici plutôt le schéma de celle de Claire d'Assise, réalisant de nombreux miracles in vita ${ }^{46}$, que de celle de Louis IX, qui n'en produit aucun.

Il est possible de dresser une typologie des miracles d'Isabelle recensés dans le texte et de la comparer à celle des procès de canonisation étudiés par André Vauchez :

\begin{tabular}{|l|l|l|l|}
\hline Types de miracles & $1201-1300$ & $1300-1417$ & $\begin{array}{l}\text { Isabelle } \\
40 \text { miracles }\end{array}$ \\
\hline \hline Résurrection & $2,2 \%$ & $10,2 \%$ & aucun \\
\hline \hline Maladies contagieuses & $28,6 \%$ & $31,2 \%$ & $42,5 \%$ \\
\hline \hline Paralysies & $28,8 \%$ & $12,5 \%$ & $2,5 \%$ \\
\hline \hline Blessures & $5,2 \%$ & $5,6 \%$ & $10,0 \%$ \\
\hline \hline Aveugles, sourds, muets & $12,4 \%$ & $11,7 \%$ & $2,5 \%$ \\
\hline \hline Maladies mentales & $10,7 \%$ & $5,1 \%$ & $2,5 \%$ \\
\hline \hline Enfantement & $1,2 \%$ & $3,3 \%$ & aucun \\
\hline \hline Délivrance, protection & $3,2 \%$ & $11,8 \%$ & $7,5 \%$ \\
\hline \hline Miracles religieux & $3,8 \%$ & $5,0 \%$ & $17,5 \%$ \\
\hline
\end{tabular}




\begin{tabular}{|l|l|l|l|}
\hline Divers & $3,9 \%$ & $3,6 \%$ & $15,0 \%$ \\
\hline
\end{tabular}

Source : A. Vauchez, La sainteté en Occident aux derniers siècles du Moyen Âge, op. cit., p. 547. résurrection ni aucun lié à l'enfantement ne sont présents, l'absence de ce dernier type étant explicable par la vie monastique. Les miracles intitulés "divers" sont exceptionnellement nombreux : ils sont de nature spécifique, liés à la virginité et aux livres. Les miracles d'Isabelle se produisent le plus souvent à l'abbaye de Longchamp, tout comme ceux de Louis IX se concentrent à Saint-Denis ${ }^{47}$. Vingt-et-une des trentecinq personnes concernées sont les religieuses de Longchamp. Les hommes miraculés sont soit des religieux liés au couvent, soit des proches de la famille royale, à deux exceptions près. Deux des quatre enfants sont également liés à la famille royale. Le miracle est le plus souvent immédiat. Si le contact de reliques ou de la tombe d'Isabelle est fréquent, dix miracles sont cependant réalisés à distance. Les miracles ne sont pas datés. Seulement trois d'entre eux comprennent une mention temporelle indiquant la proximité $d u$ décès $d^{\prime} I s a b e l l{ }^{48}$. Les miracles de saint Louis racontés par Guillaume de Saint-Pathus ${ }^{49}$ sont bien cadrés dans la société laïque et ecclésiastique du XIII siècle $^{50}$. Ceux d'Isabelle sont très spécifiquement féminins, et liés au monastère ou à la famille royale.

21 Certains traits de la Vie d'Isabelle doivent être replacés dans le cadre des modalités générales d'une sainteté féminine au XIII ${ }^{\mathrm{e}}$ siècle. Les Franciscains ont joué un grand rôle dans la promotion de cultes féminins, parmi lesquels les saintes laïques sont bien présentes ${ }^{51}$. Le thème de la souffrance corporelle, par laquelle une femme doit passer pour accéder à la sainteté, est fréquent dans les vies de ces saintes. La maladie et le jeûne sont des signes classiques de cette souffrance corporelle. Comme Claire d'Assise, la princesse Isabelle est gravement malade à de nombreuses reprises, parfois même « en peril de mourir ». Elle jeûne trois fois par semaine et refuse les mets raffinés de la $\operatorname{cour}^{52}$. Le jeûne est une constante dans le rapport des femmes à la nourriture : cette pratique alimentaire est un moyen de contrôler leur corps et leur destin ${ }^{53}$.

Pour faire pénitence, Isabelle se fait flageller de telle sorte que «sa robbe estoit souvent teinte de sang ${ }^{54}$. Les saintes mendiantes pratiquent la mortification de leur corps: Marguerite de Hongrie se flagelle souvent, provoquant de véritables plaies. Claire de Montefalco s'inflige également la discipline ${ }^{55}$. Le fait de répandre son sang est la contrepartie du sang menstruel, symbole de l'impureté du corps féminin ${ }^{56}$. Mais les femmes qui accomplissent le rite de la flagellation doivent le faire séparément et en privé $^{57}$. Le cas d'Isabelle est ambigu, puisque cette pratique est accomplie en secret par une de ses dames de compagnie, mais celle-ci en parle ensuite devant toutes les autres ${ }^{58}$. Isabelle conquiert bien sa sainteté en châtiant son corps, mais d'une manière qui est ici aux marges de ce qui est autorisé pour les femmes.

Cette souffrance est associée à la chasteté, en vue d'arriver à l'union mystique avec le Christ. Isabelle refuse le mariage prévu pour elle pour mener une vie chaste: Agnès d'Harcourt dit qu'« elle avoit esleu le perdurable espoux Nostre Seigneur Jesus-Christ, en parfaicte virginité » et plus loin que "tres devotement sa vie fina en parfaite virginité $"^{59}$. L'un des miracles posthumes d'Isabelle consiste à protéger une pucelle qui risquait de perdre sa virginité ${ }^{60}$. La virginité et la chasteté préparent la jeune fille à l'union mystique, et le jeûne et la maladie permettent son accomplissement ${ }^{61}$. Le corps 
d'Isabelle, resté chaste, achève d'être dépouillé de ses particularités sexuelles par sa "sainte anorexie $»^{62}$. C'est une forme paroxystique de l'Imitatio Christi. Isabelle, par la souffrance volontaire ou involontaire infligée à son corps, et par la préservation de la virginité en vue de l'union avec le Christ, témoigne d'une sainteté empreinte de mysticisme, comme beaucoup de saintes laïques de la fin du Moyen Âge. On retrouve dans la Vie de Claire d'Assise les mêmes caractéristiques ${ }^{63}$.

Isabelle est une princesse de sang royal. Si le type de la sainte reine disparaît au XII siècle, quelques cas sont connus au XIII $^{\mathrm{e}}$ siècle, notamment Hedwige de Silésie et Élisabeth de Hongrie ${ }^{64}$. Cette situation est tout de même rare. De plus, la vocation pousse Isabelle à prendre des décisions hors du commun, et surtout à choisir de rester vierge sans entrer pour autant dans les ordres. Isabelle s'oppose ici clairement aux obligations liées à son statut de princesse royale. Elle aurait déjà dû être mariée en 1230 à Hugues de la Marche, mais ce dernier épousa finalement Yolande, fille du comte de Bretagne $^{65}$. L'époux qui lui est destiné ensuite est Conrad de Hohenstaufen, fils de Frédéric II. Elle le refuse à l'été $1243^{66}$. Ce choix est également affirmé par l'usage fait de sa dot, qu'elle décide de distribuer : « Monseigneur le roy Louys son pere li laissa moult grand deniers quand il mourut, et tout elle donna pour Dieu $»^{67}$. Louis VIII lui a en effet laissé par son testament de juin 1225 un legs de vingt mille livres ${ }^{68}$. Ce refus du mariage s'effectue contre la volonté du roi son frère, de son entourage, et même du pape Innocent IV : celui-ci lui écrit de se marier pour « les proufits qui viennent du mariage de telle dame $»^{69}$. Mais Isabelle reste inflexible, et une autre lettre papale approuve finalement son dessein de vivre en perpétuelle virginité ${ }^{70}$.

Claire d'Assise refuse comme Isabelle deux mariages et doit affronter la vindicte familiale ${ }^{71}$. Mais Claire n'est pas une fille de roi comme Isabelle, pour qui le mariage est plus qu'un destin normal : il s'agit d'une affaire politique, qui permet d'apporter des alliances au royaume. Le refus d'Isabelle est exceptionnel. L'itinéraire de sainte Élisabeth de Hongrie, par exemple, est gouverné dès l'enfance par la politique matrimoniale de sa famille. Elle est obligée de faire un mariage dynastique et ne peut suivre sa vocation ${ }^{72}$. Marguerite de Hongrie refuse trois fois des offres de mariages royaux, mais devient religieuse en 1254 , ce que ne fait pas Isabelle ${ }^{73}$. Isabelle donne donc par sa vie un modèle de comportement marqué par une autonomie spécifique d'une princesse de la famille royale. Cette possibilité d'émancipation est peut-être liée à l'évolution des conditions de l'éducation féminine au XIII ${ }^{\mathrm{e}}$ siècle : les jeunes filles de la plus haute noblesse peuvent alors bénéficier aux côtés de leurs frères d'un enseignement particulier en latin et langue vulgaire ${ }^{74}$.

Agnès d'Harcourt insiste justement beaucoup sur le fait qu'Isabelle est lettrée. Le premier élément de sa vie à nous être présenté est son apprentissage des saintes Écritures: "[elle] apprenoit a entendre la divine Escriture [...] et quand elle fust introduicte des lettres suffisamment $[. ..] \aleph^{75}$. Agnès d'Harcourt précise que la princesse sait lire et écrire le latin, ce qui lui permet d'étudier seule les Écritures, mais aussi de corriger les lettres de ses chapelains : «elle les amendoit quand il y avoit aucun faux mot $\aleph^{76}$. Isabelle semble donc surpasser les clercs de son entourage dans la maîtrise de cette langue savante.

Tout comme Claire d'Assise rédige la règle de son ordre ${ }^{77}$, Isabelle établit elle-même celle des Clarisses de Longchamp en 1255: Agnès d'Harcourt insiste sur le travail personnel de la princesse: "elle travailla tant et estudia qu'a peine le pourroit-on raconter $\aleph^{78}$. Elle prend conseil auprès des plus grands maîtres franciscains parisiens de 
l'époque, en particulier Bonaventure : celui-ci incarne une tendance modérée en ce qui concerne la pauvreté, ce qu'il faut lier à la souplesse de la règle d'Isabelle en ce domaine. La règle choisie est en effet une forme adoucie de celle de Claire d'Assise : elle autorise les religieuses à hériter et posséder des biens en commun. Elle est approuvée par Alexandre IV en 1259, puis remaniée par Urbain IV en $1263^{79}$.

La valorisation de la culture savante dans la sainteté est un fait nouveau de la fin du $\mathrm{XIII}^{\mathrm{e}}$ et $\mathrm{du}$ début $\mathrm{du} \mathrm{xIV}^{\mathrm{e}}$ siècles. Certains textes tendent à mettre en valeur les dispositions précoces des saints à l'apprentissage des savoirs : la Vie du bienheureux Ambroise, un Dominicain siennois qui vécut de 1220 à 1286, présente ainsi un intellectuel chrétien avec un goût inné de la lecture qui le prédispose à sa future mission de prêcheur ${ }^{80}$. L'éducation des saints commence vers 1300 à tenir une place essentielle dans leurs Vies ${ }^{81}$. Cependant, si cette mise en valeur de la culture est claire chez les Dominicains, elle est moins nette chez les Franciscains. De plus, Isabelle est une femme. Ce goût du travail intellectuel est un trait exceptionnel, commun à Claire d'Assise et Isabelle de France.

Deux miracles posthumes d'Isabelle sont liés aux livres. Dans le premier de ces deux miracles, Isabelle aide à retrouver un livre perdu qui lui avait appartenu de son vivant $^{82}$. Dans le second, un bréviaire tombé dans l'eau redevient lisible par son intervention : «Il fut restauré en son premier estat, et est beau et lisable comme devant ce qu'il cheut en l'eau ${ }^{83}$. Par ces miracles, Isabelle apparaît comme une protectrice des livres et de la lecture ${ }^{84}$. Un seul manuscrit lui ayant appartenu est actuellement conservé : il s'agit d'un psautier, réalisé dans le même atelier et au même moment que le psautier de la Bibliothèque nationale de France, lat. 10525, qui appartenait à saint Louis $^{85}$. Du fait de la règle assouplie par Isabelle, les Clarisses de Longchamp ont possédé de nombreux livres, dont la liste se trouve dans les inventaires de l'abbaye conservés aux Archives nationales ${ }^{86}$. Isabelle, sainte lettrée, protectrice des livres, fréquentant les intellectuels de son temps, échappe encore une fois nettement aux cadres d'ordinaire réservés aux femmes de la famille royale.

En définitive, si la sainteté d'Isabelle obéit à certains critères "classiques » de la sainteté féminine au XIII ${ }^{\mathrm{e}}$ siècle, son comportement annonce également celui des tertiaires italiennes ${ }^{87}$. Sa vie reproduit les grands traits du parcours de Claire d'Assise, transposés dans le cadre de la famille royale de France. Son choix du célibat, bien qu'étant une princesse royale, et son goût de l'étude sont des éléments plus originaux et qui fondent à travers Isabelle un nouveau modèle de comportement féminin.

Louis IX et Isabelle : deux saintetés similaires et complémentaires

31 Agnès d'Harcourt place également Isabelle dans la continuité du parcours religieux de son frère. Quel rapport entretiennent entre eux les textes concernant saint Louis et la Vie d'Isabelle? Quel sens prend alors l'écriture de cette version religieuse et féminine d'une sainteté laïque et royale?

Louis IX, frère aîné d'Isabelle, est très présent dans le texte en tant que témoin et acteur du récit. Les femmes qui entourent Isabelle appartiennent à la clientèle de saint Louis : Pernelle de Montfort ${ }^{88}$ et Agnès d'Harcourt sont sœur et fille de seigneurs qui furent des compagnons du roi. Ce dernier participe à la fondation du monastère de Longchamp, et apparaît lors de certains miracles. Le premier miracle d'Isabelle consiste à guérir l'enfant de l'un de ses serviteurs. Agnès d'Harcourt souligne le lien fraternel entre Louis et Isabelle en précisant souvent : « le roy son frere ${ }^{89}$. Elle utilise l'épithète " saint $~{ }^{0}$ pour l'un comme l'autre alors qu'aucune canonisation officielle n'a encore eu 
lieu. La sainteté du frère est présentée comme inséparable de celle de la sœur : cette sainteté se veut bel et bien de nature familiale et dynastique par l'intermédiaire d'une sorte de couple fraternel ${ }^{91}$.

L'auteur attribue faussement à Salomon cette citation de l'Ecclésiastique: "Tant comme tu es plus grand, humilie toy en tes œuvres en toutes choses $"^{92}$. Salomon est le constructeur du Temple, comme saint Louis celui de la Sainte-Chapelle: l'un est souvent donné comme modèle de l'autre. Geoffroy de Beaulieu et Boniface VIII comparent le roi de France à Salomon non pour sa puissance, sa richesse et sa sagesse exaltées par la Bible, mais pour son caractère de rex pacificus ${ }^{93}$. Agnès d'Harcourt, en affirmant qu'Isabelle a suivi l'exemple de Salomon, fait de la princesse l'héritière spirituelle d'un modèle de roi idéal, comme son frère.

Les Franciscains jouent un rôle essentiel dans la vie d'Isabelle comme dans celle de son frère. Les confesseurs de la jeune fille sont des maitres franciscains, tel Eudes de Rosny. Agnès d'Harcourt dit qu'elle "avoit accoustumé d'avoir a confesseurs moult bonnes personnes et anciennes et maistres de divinité, et tres grande reverance leur portoit ${ }^{94}$. Une bulle du pape Innocent IV, le 26 mai 1254, autorise en effet Isabelle à garder près d'elle des frères mineurs comme directeurs de conscience ${ }^{95}$. Louis IX entretient d'étroites relations avec les frères mendiants, qui influencent son action publique. Tous ses confesseurs sont des frères mendiants, dominicains comme Geoffroy de Beaulieu ou franciscains comme Jean de Mons ${ }^{96}$.

Les maîtres qui aident la princesse à rédiger la règle de Longchamp sont tous des Franciscains. Or ils sont également en relation avec Louis IX : Bonaventure est venu à la demande du roi prononcer dix-neuf sermons dans la Sainte-Chapelle entre 1257 et $1259^{97}$. Un exemplum d'origine italienne relate également une discussion théologique entre le roi et le maître ${ }^{98}$. Lors de la querelle entre maîtres séculiers et maîtres mendiants à l'université de Paris entre 1254 et 1257, le roi appuie les décisions pontificales favorables à ces derniers. Il applique rigoureusement la sentence à l'égard de Guillaume de Saint-Amour ${ }^{99}$. Le fait que ces maîtres soient réunis pour conseiller la princesse est une preuve supplémentaire de l'appui de Louis IX et de sa famille à leur parti dans la querelle qui se déroule alors. Isabelle suit les choix religieux de son frère, donnant ainsi le sentiment d'une solidarité de la famille royale en la matière. L'influence commune des ordres mendiants se traduit par un modèle de sainteté commun.

Certains éléments du comportement religieux d'Isabelle, caractéristiques du modèle " évangélique » dominant à la fin du XIII ${ }^{e}$ siècle, rappellent en effet ceux rapportés par les hagiographes de Louis IX qui cherchent à faire de lui un "roi mendiant». Ces éléments sont des stéréotypes, mais certains traits précis, plus originaux, se retrouvent d'un texte à l'autre. La vigueur des flagellations de Louis IX effraie son entourage comme dans le cas d'Isabelle : après chaque confession, il reçoit de la main de l'un de ses confesseurs la discipline faite de cinq petites chaînes de fer. Geoffroy de Beaulieu relate que l'un d'eux aurait frappé avec une force excessive, blessant sérieusement le roi ${ }^{100}$. L'obsession de la charité marque autant le frère que la sœur. Agnès d'Harcourt relate que Blanche de Castille parvient à faire manger la princesse en promettant de donner "quarante sols aux pauvres ${ }^{101}$. Isabelle a de plus coutume, le jeudi saint, d'inviter treize pauvres, leur laver les pieds, et les servir elle-même en nourriture et en chaussures. Elle leur fait une aumône à chacun de trente sous parisis ${ }^{102}$. Cette habitude se retrouve telle quelle dans la Vie de saint Louis de Guillaume de Saint-Pathus, 
d'écriture plus tardive ${ }^{103}$. Tout comme saint Louis nourrit un moine lépreux de Royaumont, Isabelle s'occupe d'une lépreuse, la « damoiselle de Meru » ${ }^{104}$.

Avec la somme héritée de son père, Isabelle désire fonder soit un hôpital, soit un monastère de Clarisses ${ }^{105}$. Elle témoigne ainsi d'une volonté de reproduire les actes de Louis IX, mais également de s'inscrire dans un projet familial. Louis VIII a laissé à son fils une forte somme pour établir près de Paris un monastère auquel la famille royale aurait été particulièrement liée. Royaumont, affilié à l'ordre de Cîteaux, est ainsi fondé en 1228. Blanche de Castille fonde Maubuisson, abbaye de Cisterciennes, en 1241. Louis IX a fait bâtir de son vivant et par testament de nombreux édifices religieux et hospitaliers: l'église abbatiale de Saint-Antoine près de Paris, les hôtels-Dieu de Pontoise et de Vernon, la maison des aveugles de Paris ${ }^{106}$. Isabelle hésite entre ces deux types de lieux avant de fonder les Clarisses de Longchamp. Ce monastère s'inscrit dans le système de nécropoles familiales de la dynastie capétienne. Saint-Denis est le lieu d'inhumation des rois. Blanche de Castille est enterrée à Maubuisson. Les enfants royaux sont à Royaumont. Le monastère de Longchamp est destiné à devenir un autre lieu de prière pour la famille royale, où les femmes seront les gardiennes de la mémoire familiale. Isabelle tient à ce que les religieuses soient appelées " seurs mineures " dans la règle de l'abbaye ${ }^{107}$. La royale sororité d'Isabelle lui permet peut-être d'avoir accès à cette tendre appellation refusée aux femmes qui entourent Claire ${ }^{108}$.

Isabelle témoigne comme Louis IX d'une horreur physique des péchés de langue. La parole, pour elle, procède d'un souci de vérité et de pureté. La plupart du temps, elle préfère garder le silence, notamment à table, où son refus de parler n'est pas sans rapport avec son refus de s'alimenter ${ }^{109}$. L'auteur signale l'importance prise par la confession dans sa vie, une pratique rendue obligatoire par le concile de Latran en 1215 : «Moult volontiers se confessoit, et souvent aussi comme chaque jour, et moult devotement $»^{110}$. Elle veille même, scrupule excessif, à ce que son confesseur ne s'endorme pas. Le péché confessé par l'aveu s'oppose au péché de langue commis devant elle, et qu'elle ne peut tolérer : " elle ne pouvoit souffrire que l'on dict nul mal d'autruy devant li ne nulle mensonge, et en avoit si grand horreur que tout la face l'en muoit ${ }^{111}$. Ce rejet se traduit physiquement sur son visage.

Les théologiens $\mathrm{du} \mathrm{XIII}^{\mathrm{e}}$ siècle placent dans leur classification des péchés celui de bouche, le peccatum oris. Albertano de Brescia compose en 1245, à l'usage de son fils, un Ars loquendi et tacendi, art de parler et de se taire, qui a un ample succès. Le silence à table est, en particulier lors des festins des princes, une arme efficace contre la flatterie $^{112}$. Or ces péchés de langue sont également l'obsession de Louis IX qui gouverne par la parole et prêche le bon comportement. Il est le premier roi dont certains propos sont rapportés au style direct ${ }^{113}$. Il pratique assidûment la confession, comme l'en loue Geoffroy de Beaulieu ${ }^{114}$, et la recommande à son fils et à sa fille dans ses Enseignements $^{115}$. Il refuse de mentir, notamment lorsqu'il est prisonnier. Sa haine du blasphème peut être violente, et se traduit dans l'ordonnance de 1269 qui punit le blasphémateur d'une amende, du pilori ou du fouet ${ }^{116}$.

Ces différents éléments témoignent $d u$ fait $q u$ 'Isabelle reproduit dans son comportement religieux, dans ses œuvres de miséricorde et dans ses actes de piété, le modèle de son frère. Les vertus de Louis IX sont partagées par sa sœur. Agnès d'Harcourt cherche ainsi à montrer qu'Isabelle est aussi digne que Louis IX de faire l'objet d'une enquête de canonisation. 
Isabelle a recours à l'aide de son frère, roi de France, pour la fondation des Clarisses de Longchamp. Elle laisse Louis IX se charger des relations avec la papauté : «elle [le] faisoit chevetin de toutes ses besognes, et il le faisoit moult courtoisement, et envoyoit les lettres et les propres messages $»^{117}$. Reconnaissante, elle s'incline devant lui à cette occasion, à la grande gêne du roi. Au mois d'avril 1255 , le roi charge son chapelain, le frère Mathieu, et Simon Duval Grimon d'acquérir quatre arpents de terre à Saint-Cloud pour construire le monastère. En mai, il en achète encore quatre aux autorités de Suresnes. Le 10 juin 1256, il pose la première pierre de l'édifice. La construction terminée en 1259 a coûté trente mille livres ${ }^{118}$. Le roi a donc secondé non seulement diplomatiquement, mais aussi financièrement sa sœur. Agnès d'Harcourt a peut-être tendance à réduire la participation du roi à la fondation d'Isabelle pour mieux mettre en valeur le rôle de cette dernière. Cependant, Isabelle ne fait pas toujours preuve de soumission à l'égard du roi : ainsi, lorsque celui-ci lui demande de lui donner le couvrechef qu'elle a tissé, elle refuse et le remet « secretement a une pauvre femme qui gisoit en grand langueur ${ }^{119}$. Isabelle ment et désobéit pour un motif de charité.

Agnès d'Harcourt tend de surcroît à montrer que la sainteté d'Isabelle est plus parfaite que celle de saint Louis. La princesse parvient à mener toute sa vie en parfaite virginité, ce qui n'est pas le cas de Louis IX, marié à Marguerite de Provence. Il respecte, certes, les périodes d'interdits en matière de relations charnelles ${ }^{120}$, mais doit lutter contre sa chair qui n'en est pas moins faible ${ }^{121}$. Le roi a dû se plier à l'obligation dynastique, alors qu'Isabelle a réussi à s'y soustraire. De plus, la princesse possède le don des larmes. Agnès d'Harcourt la dépeint en pleurs à la fin de ses prières : elle " estoit souvent en grande abondance de larmes, si que, quand elle issoit de son oratoire, elle avoit les yeux si enflez et si rouges qu'il aparoit bien que merveilleusement avoit espandues des larmes ${ }^{122}$. Les larmes représentent au XIII ${ }^{\mathrm{e}}$ siècle une étape essentielle vers la sainteté. C'est l'expression de la grâce de Dieu accordée au pécheur. Ainsi Umiliana dei Cerchi, à Florence au milieu du XIII ${ }^{\mathrm{e}}$ siècle, se met-elle de la chaux vive sur les yeux pour se faire pleurer ${ }^{123}$. Ce signe est parfois refusé à saint Louis : il arrive que le roi ne parvienne pas à pleurer et qu'il se plaigne de $l^{\prime}$ «aridité et la dureté de son cœur »124. Isabelle pleure, elle, en abondance, comme Claire d'Assise ${ }^{125}$. Louis IX est roi de France: Isabelle, seulement princesse. Pour démontrer sa sainteté, Agnès d'Harcourt décrit une princesse réalisant ce que Louis IX n'a pu parfaitement accomplir.

Trois miracles intégrés à la Vie d'Isabelle sont réalisés par saint Louis grâce à l'intercession de sa sœur. Lorsque sœur Julienne perd un livre, Isabelle lui apparaît dans son sommeil et lui conseille de faire appel à saint Louis : "qu'elle en requit monseigneur le roy sainct Louys son frere ». Après une prière à ce dernier et une offrande de cire, le livre est retrouvé ${ }^{126}$. Le livre est un objet important pour la sainteté de Louis IX : durant sa captivité en Égypte, le roi retrouve à côté de lui son bréviaire que Dieu lui a fait miraculeusement apporter ${ }^{127}$. Mais le roi n'est pas actif dans la réalisation de ce miracle, contrairement à sa sœur. Sœur Jeanne de Louveciennes fait ensuite appel à Isabelle pour la soigner. La princesse lui apparait en songe, et lui dit: "Allez a mon frere». Jeanne voit la tombe du roi à Saint-Denis, et Isabelle demande à Louis IX de la guérir ${ }^{128}$. Enfin, la même sœur Jeanne de Louveciennes, malade et portée sur la tombe d'Isabelle, voit le spectre de cette dernière venir s'asseoir à côté d'elle sur la tombe et lui dire à nouveau : «Allez a mon frere». Cette fois, la sœur ne voit pas seulement saint Louis mais « une procession de roys mout noblement appareillez et 
tous couronnez ", le saint roi fermant la procession. La sœur est à nouveau guérie par son action ${ }^{129}$.

\section{faire des miracles. La représentation de la relation d'Isabelle et de son frère dans l'au-} delà emprunte ici au thème de la " cour céleste ", selon lequel les hiérarchies terrestres sont reproduites dans les sphères célestes ${ }^{130}$. Guillaume de Saint-Pathus cite une phrase qu'aurait dite saint Louis à propos de l'intercession des saints qui semble s'appliquer à ce type de représentation : «Il en est par similitude des saints du paradis comme il en est des conseillers des rois. Les saints du paradis, étant les intimes de Notre Seigneur et ses familiers, peuvent l'invoquer en toute assurance $\aleph^{131}$. Dans le texte d'Agnès d'Harcourt, saint Louis est présenté comme supérieur en puissance à Isabelle. La princesse fait le lien entre les fidèles et le roi, intermédiaire et avocate essentielle à l'apparition de ce dernier et à la réalisation du miracle. Le troisième récit la place même en intercesseur de la lignée des rois de France, présentée comme sainte dans son ensemble. L'image de cette procession pourrait être inspirée de la Galerie des rois de Juda sur la façade de la cathédrale Notre-Dame de Paris ${ }^{132}$.

L'œuvre d'Agnès d'Harcourt entretient, en définitive, des relations complexes avec la série des premières biographies de saint Louis. Si le plan du récit de la vie d'Isabelle ressemble à celui utilisé par Geoffroy de Beaulieu, la présence de miracles rappelle plutôt la démarche de Guillaume de Chartres ${ }^{133}$. Des thèmes communs sont utilisés dans tous ces textes, mais des exemples précis se retrouvent plus particulièrement de la Vie d'Isabelle à l'œuvre de Guillaume de Saint-Pathus. Trois miracles d'Isabelle constituent également de nouveaux miracles de saint Louis, qui ne sont pas cités par ses biographes. La démonstration de la sainteté d'Isabelle vient donc appuyer celle du roi de France, en imitant et en prolongeant l'œuvre de ses premiers biographes. Le monastère de Longchamp, lieu de miracles conjoints d'Isabelle et du roi de France, devient un nouveau lieu de mémoire pour la famille royale.

Quel écho et quel succès la Vie d'Isabelle a-t-elle pu rencontrer? Isabelle n'a jamais été canonisée, à la différence de son frère. Elle n'est béatifiée qu'en 1521 par la bulle Piis omnium de Léon $\mathrm{X}^{134}$. Le monastère de Longchamp a accueilli comme religieuses d'autres princesses : Blanche, fille de saint Louis, y est pensionnaire avant son mariage ; Blanche, fille de Philippe V, y revêt l'habit de nonne en 1318, et Jeanne, fille de Jeanne de France reine de Navarre, en $1337^{135}$. Philippe V vient y mourir en 1322. De nombreux membres de la famille royale rendent visite aux religieuses ou leur font des legs. La sainteté d'Isabelle a-t-elle pu être un modèle pour les princesses? Si le texte de la Vie est apparemment peu copié ${ }^{136}$, il est sûrement lu à Longchamp, aux nonnes comme aux invités. Le culte d'Isabelle s'est développé au monastère à partir de $1517^{137}$. La Vie a servi de base au $\mathrm{XV}^{\mathrm{e}}$ siècle pour la rédaction de l'épitaphe d'Isabelle ${ }^{138}$, et pour celle d'un office utilisé à Longchamp au XVI ${ }^{\mathrm{e}}$ siècle $^{139}$.

(s) fondé en 1270 à Troyes un monastère de Cordelières suivant la règle de Longchamp ${ }^{140}$. La règle d'Isabelle, reprise par Urbain IV et permettant aux religieuses de posséder des biens et d'en hériter, a connu un grand succès au sein de l'ordre des Clarisses. Elle est adoptée dans de nombreux monastères de "riches Clarisses » liés à la famille royale ${ }^{141}$ : par exemple, outre celui de Troyes, ceux de Provins ${ }^{142}$ et Toulouse143.

Isabelle de France choisit, en refusant le mariage, un destin hors du commun pour une princesse capétienne. Au-delà des topoï hagiographiques, la vierge savante emprunte à 
la fois à un modèle féminin de sainteté proche de Claire d'Assise, et à un modèle royal et masculin, celui de saint Louis. Cette Vie d'Isabelle, texte écrit par une femme à propos d'une sainte, est en même temps une contribution supplémentaire et originale en faveur de la canonisation de Louis IX et de la sainteté dynastique capétienne.

Édition de la Vie d'Isabelle de France par Agnès d'Harcourt

Au moins trois manuscrits de la Vie d'Isabelle de France écrite par Agnès d'Harcourt sont attestés à la fin du Moyen Âge :

50 - Le plus ancien manuscrit du texte, réalisé à la fin du XIII siècle, est mentionné en 1325 dans les inventaires de l'abbaye de Longchamp ${ }^{143}$. Il n'apparaît pas dans les inventaires $\mathrm{du} \mathrm{Xv}^{\mathrm{e}}$ siècle, sans doute à cause de sa forme de rouleau. Il se trouve pourtant toujours au monastère en 1699: Pierre Perrier affirme qu'il fait partie des pièces relatives à Isabelle que les sœurs de Longchamp lui ont montré, et le décrit de la façon suivante: "C'est un volume roulé à l'ancienne manière, et composé de huit feuilles de parchemin cousues bout à bout, long d'environ sept pieds, et large de 8 à 11 pouces sur la longueur. La première feuille est d'une écriture plus récente que les autres. [...] Ainsi la première feuille est perdue, laquelle on a transcrite sur celle-ci, qui contient une espèce de préface, qui est telle: "Nous avons proposé d'écrire la vie de nostre sainte et benoite dame et mere..." " ${ }^{144}$. Le manuscrit est encore à Longchamp en 1741 , lorsque le R. P. Stephanus Souciet, qui le dit autographe, atteste qu'il est du siècle de saint Louis et écrit sur du parchemin, non en librum mais en volumen ${ }^{145}$. Il a dû rester à l'abbaye jusqu'à la confiscation des biens de celle-ci en $1792^{146}$. Il est aujourd'hui perdu ${ }^{147}$.

51 - Le second exemplaire du texte est cité comme étant relié avec la règle de Longchamp en $1325^{148}$, comme l'est une Vie de sainte Claire en français à la suite d'un second exemplaire de la même règle à partir de $1448^{149}$. Ce manuscrit est conservé à l'abbaye de Longchamp, comme en témoignent les inventaires des XIVe-XVe siècles, jusqu'en 1483. Aucun indice ne permet de dire s'il est resté à l'abbaye comme le précédent jusqu'en 1792, ou bien s'il en est sorti avant, du fait de l'absence d'inventaires détaillés postérieurs au $\mathrm{Xv}^{\mathrm{e}}$ siècle. Il est en tout cas également perdu aujourd'hui.

- Les inventaires de la librairie royale du Louvre de 1373 à 1424 portent la mention d'un troisième manuscrit. Il est décrit de la manière suivante en 1373 : «La vie suer Ysabeau de Longchamp, qui fu suer saint Loys, et ses miracles; une histoire d'une nonnain au commencement. $»^{150}$, et de façon un peu plus détaillée en $1423:$ «Item. La Vie suer Isabeau de Longchamp, qui fut seur saint Loys, et ses miracles; escript en François de lettre de forme à une coulombe, et une Histoire d'une nonnain, au commencement. Comm ${ }^{t}$ ou $\mathrm{II}^{\mathrm{e}}$ fueillet "Seigneur Jhesus Crist" et ou derrenier “...guement travaillé" en ung petit livret couvert de cuir rouge, sanz emprainte, à deux fermouers de laton...V. s. p. " ${ }^{151}$. Il n'est pas non plus identifiable actuellement.

La perte des manuscrits médiévaux explique la rareté des études portant sur le texte de la Vie d'Isabelle. Deux copies modernes permettent cependant de connaître de manière assez exacte ce document d'une extrême richesse. La première se trouve dans un cahier de notes de Sébastien Le Nain de Tillemont, et date de $1653^{152}$. Le copiste, Antoine Le Maistre, affirme avoir travaillé d'après le manuscrit conservé à Longchamp sous forme de rouleau ${ }^{153}$. La deuxième copie moderne de la Vie d'Isabelle de France est donnée par Charles Du Fresne, sieur Du Cange, en 1668 ${ }^{154}$. Du Cange dit avoir copié un exemplaire 
fourni par Antoine Vyon d'Hérouval ${ }^{155}$. Malheureusement il ne donne pas plus d'indications au sujet de ce manuscrit. in le lignage, encore fust elle plus haute et plus noble de mœurs. Elle sçavoit bien que icelle seule est la vraye noblesse qui est ornement de l'ame par bonté de l'ame et par saincte vie, si comme il ${ }^{171}$ appaira cy aprés. Elle fust fille et espouse et speciale amie de Nostre Seigneur Jesus Christ, et tous ses desirs, et toute l'intention ${ }^{172}$, et tous ses labeurs si furent de destruire pechez et de planter vertus en soy et en autruy. Elle fust mirouër d'innocence, exemplaire de penitence, rose de patience, lis de chasteté, fontaine de misericorde. Elle fust escolle de toutes bonnes mœurs, car elle fust escoliere speciale de l'escolle de Nostre Seigneur Jesus Christ $^{173}$, qui dit a ses disciples: "Approchez, apprenez de moy que je suis doux et debonnaire et humble de cœur $»^{174}$. Icelle leçon retint bien especiaument nostre benoiste et saincte et noble dame et mere madame Isabelle de France ${ }^{175}$. En toutes ses œuvres n'apparoist fors humilité de cœur et debonnaire, selon ${ }^{176}$ que Salomon enseigne : «Tant comme tu es plus grand, humilie toy en tes œuvres ${ }^{177}$ en toutes choses $»^{178}$. 
61 [5] Ceste ${ }^{179}$ benoiste et excellente dame en sa jeunesse tres volontiers demeuroit en la chambre, et apprenoit a entendre la divine Escriture, et ne vouloit aller es esbatemens ${ }^{180}$ la ou les femmes de ses freres et les autres dames alloient. Et quand elle fust introduicte des lettres suffisamment, elle s'estudioit a apprendre et ouvrer de soye, et faisoit estolles et autres paremens a saincte Eglise. Et quand on lui apportoit images de Nostre Seigneur ou de Nostre Dame, elle les recevoit si joyeusement que ce estoit merveilles, et monstroit bien qu'elle les aymoit mieux et avoit plus [p. 170] chers que nul autre present d'ornement que l'on ly peut faire.

62 [6] $\mathrm{Au}$ temps de sa jeunesse, quand madame la reine Blanche ${ }^{181}$ vivoit, qui merveilleusement l'aimoit tendrement, et faisoit orner son corps de moult beaux et haults ornemens et de riches, elle me dit de sa bouche qu'elle avoit aussi bon cœur et aussi devot a Nostre Seigneur quand elle avoit ces riches ornemens en son chef et en son corps, comme elle avoit quand elle eust habit plus religieux ${ }^{182}$. Et croy qu'il y en aura des autres qui bien le temoigneront si besoing en est. Et ceste chose monstroit bien que son cœur estoit toujours bien attentif a aymer Nostre Seigneur, et que l'amour de son cœur n'estoit pas aux ornemens ne a la gloire de ce chetif monde.

63 [7] Elle fut conjuree ${ }^{183}$ de ses amys ${ }^{184}$ a prendre a mariage au fils de l'empereur de Rome $^{185}$, qui devoit estre heritier de l'Empire, mais onques ${ }^{186}$ au mariage corporel ne s'en vout assentir, car elle avoit esleu le perdurable espoux Nostre Seigneur Jesus Christ en parfaicte virginité. Monseigneur le pape Innocent $\mathrm{IV}^{187}$ ly escrit et la prescha merveilleusement $\mathrm{de}^{188} \mathrm{si}$ marier pour les proufits qui viennent du mariage de telle

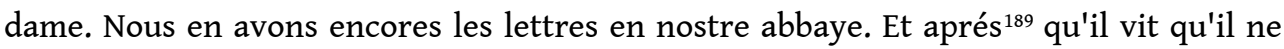
pouvoit son bon propos müer, il $\mathrm{y}^{190}$ escrit une autre lettre par laquelle il s'efforçoit

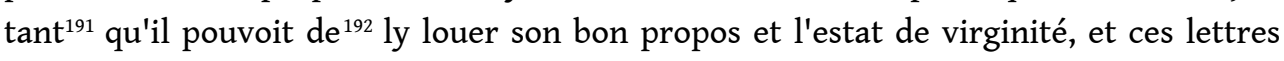
memes avons nous en nostre abbaye ${ }^{193}$.

64 [8] Elle avoit trop durement ${ }^{194}$ beau chef et reluisant, pour neant fust ce [...] ${ }^{195}$. Et quand l'on ly peignoit, ses damoiselles prenoient les ${ }^{196}$ cheveux qui li cheoient et les gardoient moult soigneusement, si que un jour elle leur demanda pourquoy elles faisoient cé197, et elles respondirent : «Madame, nous les gardons, pour ce que quand vous serez saincte, nous les garderons comme reliques ». Elle s'en rioit, et tournoit tout au neant ${ }^{198}$, et tenoit a folie ces choses. Je, sœur Agnes de Harecourt, ouÿ ces choses de la bouche a ces damoiselles qui la servoient, et encore ay je de ses cheveux de sa jeunesse.

65 [9] Il avint en sa jeunesse une trop grande maladie aiguë la prist, et, au commencement de la maladie, il convint madame la reine Blanche sa mere aller loing une journee ou deux pour les besognes du royaume, et la laissa a Saint Germain en Laye, et madame la reine Marguerite ${ }^{199}$ avec li. Et tantost la maladie engrega ${ }^{200}$ si fortement que l'on n'y attendoit aussi comme point de vie, et on s'en alla ${ }^{201}$ querre madame sa mere et monseigneur le roy son frere ${ }^{202}$ en grand haste. Et quand elle vint la, elle la trouva moult malade et en peril de mourir, de quoy elle fust moult atteinte de mesaise ${ }^{203}$ de son cœur comme mere. Elle envoya soigneusement partout pour requerre oraison, et especiaument en Angleterre ${ }^{204}$, mesmement a une personne moult religieuse et moult contemplative ${ }^{205}$, a qui elle monstroit moult a certes la mesaise de son cœur, pour ce que celle personne contraignit plus atteignement ${ }^{206}$ Nostre Seigneur par oraison pour madame ${ }^{207}$ sa fille. Et celle personne ly manda par escript que sa fille repasseroit de ceste maladie, mais fust elle certaine que jamais son cœur ne seroit au monde ne aux choses du monde. Et il y apparut bien, car oncques puis elle ne mit sus son corps nul de 
ces riches ornemens, mais, de jour en jour et de plus en plus, elle se donnoit du tout a oraison, et a œuvres de perfection, et en vie religieuse. Et de robbes, et de livree, et de toutes les choses qu'il ly convenoit a son corps a orner, elle desprisoit toutes richesses corporelles pour aquerre a l'ame de li ornement de vertu et d'humilité.

[10] Ceste benoiste et excellente dame avoit si grand amour a pureté et a innocence dés s'enfance que a peine le pourroit on raconter, si comme l'on le peust apertement ${ }^{208}$ congnoistre en toutes ses œuvres. Elle ne pouvoit souffrire que l'on dict nul mal d'autruy devant li ne nulle mensonge, et en avoit si grand horreur que tout la face l'en muoit. Si qu'il advenoit aucunes fois que, quand aucunes personnes venoient a ly ${ }^{209}$ demander l'aumosne ou pour aucunes besognes, elle envoyoit a eux avant qu'ils vinssent devant ly, et leur fai-[p. 171]-soit dire qu'ils se prinssent bien garde qu'ils ne disent fors ${ }^{210}$ que verité et que, s'elle appercevoit qu'ils disent verité, elle feroit plus volontiers ce que ils ly requerroient. Je, seur Agnes de Harecourt, porte tesmoignage de ceste chose, qui aucune fois fis ce message.

67 [11] Et en s'enfance elle estoit si accoustumee a oraison que vis ${ }^{211}$ desoubs la couverture de lict estoit elle en oraison accoutee ${ }^{212}$ et a genoux, et se repousoit dessous sa couverture. Si i $1^{213}$ avint un matin qu'ils devoient heirer ${ }^{214}$, que ciz qui devoient ${ }^{215}$ trousser ${ }^{216}$ et emmaler les licts et les robbes embrassa la couverture et la robbe, qu'il cuidoit que la robbe fust ainsi entortillee dedans le lict. Et c'estoit nostre benoiste dame et saincte mere madame Isabel qui estoit illecque ${ }^{217}$ accosté et a genoux en oraison. Et quand il vint prendre la robbe, elle s'escria si haut que les dames y accoururent, et celi fut tout esbahi et tout espouvanté. Je, seur Agnes de Harecourt, oÿ ceste chose de la bouche monseigneur le roy sainct Louys ${ }^{218}$ qui le nous raconta, et Mehaut de Godarville ${ }^{219}$ qui fust en son service ouÿ ceste mesme chose de la bouche madame Heluis de Buisemont qui avoit esté avec madame dés son enfance.

[12] Icelle mesme madame Heluis disoit qu'elle avoit veü de ${ }^{220}$ dix neuf ans que ceste benoiste dame ne mangea onques son soul de pain. Et icelle dame Heluis recordoit que madame la reine Blanche, sa mere, li disoit que, s'elle mangeoit un seul morsel, elle dourroit ${ }^{221}$ quarante sols aux pauvres, et aussi, pour parler une seule parole a monseigneur le roy son frere, elle lui promettoit aucune fois quarante sols a donner aux pauvres. Et moult de fois, elle ne le vouloit pas faire pour chose qu'elle promit pour l'amour qu'elle avoit a l'abstinence et a silence. En sa jeunesse, elle jeunoit trois jours en la sepmaine, et quand venoit a l'heure de manger, elle mangeoit si tres petit que nul corps humain ne peust en estre soustenu, si la grace de Dieu ne le fist. Et souventes fois quand elle avoit tout jour jeusné, sa viande ${ }^{222}$ estoit un peu de poiree ${ }^{223}$ et de pois baieus $^{224}$. Elle estoit servie d'assez de mets et de bonnes viandes si comme il offroit a telle dame, et tout envoyoit a l'aumosne et es enfermeries de jens de religion. Et du pire elle mangeoit et tres petit, et a chascun morsel qu'elle mangeoit, elle en mettoit dix a l'aumosne pour Dieu. Et presque tout son manger elle estoit en oraison et en silence. Elle seoit merveilleusement petit a la table, si que souvent elle se levoit avant que ses femmes qui la servoient, et rendoit graces si devotement et si ententivement que c'estoit merveille.

69 [13] Elle faisoit dire le divin office ${ }^{225}$ moult devotement et moult ententivement. Elle se levoit pour dire ses matines grand piece devant le jour, et ne se recouchoit point, et estoit continuement en oraison jusques a hault midy. Et souvente fois elle faisoit ceux qui la servoient manger avant que ly, pour estre plus longuement en oraison. Elle ne parloit point quand elle disoit ses heurs, ne devant prime, ne puis qu'elle avoit dict 
complie. S'elle n'estoit malade, elle estoit merveilleusement en oraison en Caresme plus qu'en autre temps, et estoit souvent en grande abondance de larmes, si que, quand elle issoit de son oratoire, elle avoit les yeux si enflez et si rouges qu'il aparoit bien que merveilleusement avoit espandues des larmes. Elle avoit accoustumé a estre en auraison en son oratoire jusques a l'heure du haut midy, et adonc elle issoit de son oratoire et entroit en sa chambre, et illec estoit jusques a none en estude des sainctes Escritures si comme de la Bible et des saincts Evangiles et des autres vies des saincts, car elle entendoit moult bien latin. Et si bien l'entendoit que, quand les chapelains ly avoient escrites ses lettres qu'elle faisoit faire en latin et ils ly apportoient, elle les amendoit quand il y avoit aucun faux mot, et je, seur Agnes de Harecourt, veü ceste chose plusieurs fois et autres personnes aussi.

70 [14] Merveilleusement oyoit la parole Nostre Seigneur et souvent la faisoit dire devant ly. Elle estoit de moult tendre conscience et de moult bonne. Moult volontiers se confessoit, et souvent aussi comme chacun jour, et moult devotement, et avoit accoustumé d'avoir a confesseurs moult bonnes personnes et anciennes et maistres de divinité, et tres grande reve-[p. 172]-rance leur portoit. Et quand elle se confessoit, elle se confessoit en sa capelle ${ }^{226}$, et faisoit moult reverement asseoir son confesseur devant ly, pour ce qu'elle veist qu'il fust bien ententif a ouïr sa confession, et qu'il n'entendist a autre chose et qu'il ne sommeillast. Ces choses elle m'a dit de sa bouche. Et autrement elle ne fust pas en paix de conscience s'elle ne fust certaine qu'il eust bien entendu ses pechez. Et moult tres humblement elle se tenoit devant son confesseur quand elle se confessoit, et aussi en tous autres temps ${ }^{227}$. Et moult estoit obediente a luy, pour niant fut une dame de religion ${ }^{228}$. Et avoit accoustumé quand elle se confessoit que tousjours avoit une dame et une damoiselle un peu loing de ly en telle ${ }^{229}$ disposition qu'elles pouvoient voir le confesseur et ly quand elle se confessoit.

71 [15] $\mathrm{Et}^{230}$ souvent prenoit de moult grandes disciplines, lesquelles madame Heluis, de qui nous [avons] dessus parlee, qui longuement avoit esté avec ly, dont elle se fioit moult, ly donnont ${ }^{231}$ moult secrettement. Icelle madame Heluis, quand elle la voyoit devestie, disoit devant plusieurs dames : « Vos disciplines n'estoient pas comme autres, elles estoient jusques au sang». Elle prenoit ses disciplines, non pas sans plus de simples verges, mais de fracon ${ }^{232}$ dont sa robbe estoit souvent teinte de sang.

72 [16] Ceste benoiste dame visitoit humblement et charitablement en sa propre personne les malades, et les confortoit de ses sainctes paroles, et les ammonestoit du salut de leurs ames, et les servoit de ses propres mains, et leur envoyoit largement de ses biens, et moult longuement se seoit devant eux et tastoit leur poulx. Moult avoit grande pitié de ceux qui estoient en affliction, et avoit tres grande jalousie du salut des ames.

73 [17] Pour tout le monde elle n'eust ${ }^{233}$ dict une fausse parole a esciant. Nul serment je n'oï oncques issir de sa bouche. Quand elle avoit dict une parole, c'estoit sans rappeller, pour rien elle ne fist encontre. Moult s'estudioit d'accomplir les paroles de l'Evangile, especiemment par les ${ }^{234}$ œuvres de misericorde, dont Nostre Seigneur dict ${ }^{235}$ qu'il se loera au general Jugement.

74 [18] Par grand temps, aprés ce qu'elle avoit ouÿ son office avant qu'elle disnat, elle faisoit venir grand multitude de pauvres, si que sa chambre en estoit toute environnee, et les servoit de ses propres ${ }^{236}$ mains de pain, de vin, et de potage et de pitance, et moult se travailloit ${ }^{237}$ a ces choses faire. Les grandes multitudes des aumosnes privees, qu'elle faisoit et aux religieux et aux seculiers, tant en y a qu'on ne les pourroit raconter. Une 
damoiselle, bien jentille femme, qui estoit appellee la damoiselle de Meru ${ }^{238}$, estoit en une maladrerie pres de ly ${ }^{239}$, laquelle estoit merveilleusement deffaicte ${ }^{240}$. Madame en avoit tres grande pitié, et estoit tres diligente de faire ce que besoing i $\mathrm{il}^{241}$ estoit, et li envoyoit les viandes de sa table, et eslisoit de ses mains celles qu'elle pensoit qui meilleures li estoient et plus delicieuses ${ }^{242}$, si diligemment que pour neant fust elle sa fille $^{243}$, et semblables choses fist elle plusieurs fois.

[19] Elle fila de ses propres mains un couvrechef ${ }^{244}$, lequel le sainct roy Louys son frere li demanda et li pria moult gratieusement qu'elle li donnast, et il le mettroit de nuict sur son chef. Elle ne li voulut donner si comme je, seur Agnes de Harecourt, qui estois presente, l'ouÿ de sa bouche de mes aureilles. Elle respondit au roy et li dict: «Je propose qu'il soit donné a Nostre Seigneur, car c'est le premier que je filasse oncques ». Et il li pria et dict: «Sœur, or vous prie je que vous en filiez un autre que j'aye », et elle respondit : «Je le veux bien si en file plus ». Et ce couvrechef elle envoya secretement a une pauvre femme qui gisoit en grand langueur, laquelle elle visitoit tres soigneusement chascun jour des grands benefices de sa table, et d'especialles precieuses ${ }^{245}$ viandes. Dame Jeanne et dame Peironnelle de Montfort ${ }^{246}$ entendirent ceste chose de ce couvrechef, et allerent a la pauvre femme secretement, et l'achepterent et li en donnerent tant comme elle voulut prendre, et est aux nonnains de Sainct Anthoine ${ }^{247}$, et le garderent comme reliques.

[20] Monsieur ${ }^{248}$ le roy Louys son pere ${ }^{249}$ li laissa moult grand deniers quand il mourut ${ }^{250}$, et tout elle donna pour Dieu, et especiamment elle envoya dix chevaliers outre mer. Elle assena ${ }^{251}$ tant de [p. 173] personnes en religion, que nous n'en sçavons nul nombre.

[21] Moult faisoit de biens et d'aumosnes a vefves femmes et a orfelins, et merveilleusement avoit grand compassion des gens qui estoient a mesaise et en affliction. Elle avoit ceste coustume le Jeudy absolu ${ }^{252}$ qu'elle prenoit XIII pauvres, et leur lavoit leurs pieds, et les servoit de ses propres mains de deux paires de mets, et leur donnoit soulier, et offroit a chascun XXX [sous] parisis en remembrance du prix que Nostre Seigneur fust vendu.

[22] Moult estoit en grand estude de faire chose qui pleust a Nostre Seigneur, et eut moult grande volonté de faire un hospital, et ne sçavoit lequel elle deust faire ${ }^{253}$, ou une maison de nostre ordre, ou un hospital. Elle envoya au chancelier de Paris, et li fit demander secretement lequel il cuidoit qui plairoit plus a Dieu, ou qu'elle fondast un hospital, ou une maison des sœurs mineures. Li chancelier Hemery ${ }^{254}$, qui estoit moult preudhomme et maistre de divinité, et adonc estoit son confesseur, li manda que ce n'estoit mie comparaison de l'hospital au regard de faire maison de religion especiemment de cet ordre, car la divine louange de Nostre Seigneur y est faite et celebree, et virginité y est gardee et moutepliee, et avec ce les œuvres de misericorde y sont faites, car les seurs servent ${ }^{255}$ l'une l'autre. Et dict encore au messaige : «Dictes li qu'elle ne demande plus conseil de cette chose, mais fasse la maison de religion ».

79 [23] Et tantost aprés elle fonda nostre abbaye, laquelle qui ${ }^{256}$ cousta bien XXX mille livres de Parisis. Elle fust tres diligente de la reigle qu'elle fust bonne et seure, et la fit esprouver par freres mineurs, qui estoient personnes bonnes et esprouvees et maistres de divinité si comme frere Bonnaventure ${ }^{257}$, frere Guillaume de Milletonne ${ }^{258}$, et frere Eude de Roni ${ }^{259}$, et frere Geoffroy de Vierson ${ }^{260}$, frere Guillaume de Harcombour ${ }^{261}$. Et fit mettre en la riule ${ }^{262}$ ce qui estoit es privileges, et ce qui estoit doutable et perilleux en la riule elle fit oster. Et estoit en si grand estude de ceste chose qu'elle en veilloit grande 
partie des nuicts et des jours. Elle y travailla tant et estudia qu'a peine le pourroit on raconter. Plusieurs personnes estoient en sa chambre, desquels aucuns lisoient les privileges et les autres notoient. Et estoient toujours illec ${ }^{263}$ freres mineurs, maistres de divinité, pour examiner les choses devant li en sa presence. Et tant estoit en grand soing que rien ne passast qui fust perilleux aux ames si que c'estoit merveille, et de ceste chose elle estoit en si grand soing et en si grand estude que a peine pouvoit elle reposer. Et merveilleusement avoit grand desire que ceste chose fust confirmee du pape $^{264}$.

[24] Et sur toutes choses elle vouloit que les seurs de l'abbaye fussent appellees seurs mineures, et en nulle maniere la riule ne luy pouvoit suffire si ce nom n'y fust mis ${ }^{265}$. Son benoist cœur elle eust ${ }^{266}$ a mettre en l'abbaye ce benoist nom, auquel le ${ }^{267}$ Nostre Seigneur Jesus Christ eslut Nostre Dame a estre sa mere, c'est le nom de l'humilité Nostre Dame ${ }^{268}$ qu'elle mit nom a s'abbaye, et de ce nom elle voulut qu'elle fust nommee. Et je, seur Agnes de Harecourt, li demandat : « Dame, dictes moy pour Dieu, si vous plaist, pourquoy vous avez mis ce nom en nostre ${ }^{269}$ abbaye ». Elle me respondit : «Pour ce que je n'ouÿ oncques parler de nulle personne qui le prit, dont je m'emerveille qui me semble qu'ils ont laissé le plus haut nom et le meilleur qu'ils peussent prendre, et si est le nom auquel ${ }^{270}$ Nostre Seigneur eslut Nostre Dame a estre sa mere, et pour ce l'aye je pris a mettre a ma maison ».

[25] Elle fust malade de grande maladie ${ }^{271}$ avant que la riule fust confirmee, qu'elle estoit aussi comme en langueur de cœur jusques adonc que ceste chose fust accomplie. Par grand sens et par grande humilité, elle ne vouloit rien requerre a l'Apostole ne escrire pour chose qui appartenist a sa riule ne a s'abbaye, et non faisoit elle non plus ${ }^{272}$ de nulle grande besogne qu'elle eust a faire, mais toutes ces choses elle faisoit requerir par monseigneur le roy ${ }^{273}$ son frere qu'elle faisoit chevetin ${ }^{274}$ de toutes ses besognes. Et il le faisoit moult courtoisement, et envoyoit les lettres et les ${ }^{275}$ propres messages. Et celle coustume elle avoit que, quand son sainct frere le roy Louys venoit en lieu ou elle estoit, elle l'alloit saluer et s'enge-[p. 174]-nouilloit devant li de la grande reverence qu'elle avoit a li. Et il la relevoit par les mains et li blasmoit. $\mathrm{Ce}^{276} \mathrm{li}$ desplaisoit moult, ce paroit, mais elle n'en vouloit rien laisser.

[26] Merveilleusement parloit petit et moult tenoit de silence. Et quand elle parloit, $c^{\prime}$ estoit mout priement ${ }^{277}$ et mout apenseement ${ }^{278}$. Et aucune fois frere Eude de Roni son confesseur li disoit: "Dame, il faut ${ }^{279}$ bien que vous partissiez ${ }^{280}$ et que vous vous esbatissiez, il ne depleust pas a Nostre Seigneur si vous prissiez un peu de recreation ", et li demandoit pourquoy elle tenoit tant silence. Elle li disoit ${ }^{281}$ : pour ce qu'elle avoit aucune fois trop parlé et dict de ${ }^{282}$ paroles oiseuses, si estoit bon qu'elle en fist la penitence. Mout avoit de parlemens a son confesseur des biens de vie perpetuele et des divines Escritures. Mout avoit grand reverence a Nostre Seigneur et mout le craignoit si comme elle me conta une fois secretement a moy et a li que, quand elle estoit revenue de sa chapelle d'oraison et elle estoit sur son lict appuiee, il li remembra des jugemens Nostre Seigneur. Elle me dict qu'elle trembloit si fort que la robbe et le feure ${ }^{283}$ trembloit desous li forment.

[27] Et aucunes fois vis je que d'aucunes choses qui li desplaisoient, elle blasmoit forment aucunes personnes devant moy, seur Agnes de Harecourt. Et ce pourquoy elle les blasmoit si estoit pour aucunes bonnes œuvres qu'ils ${ }^{284} \mathrm{n}^{\prime}$ avoient pas faites qu'elle leur avoit enchargees. Et pour ce qui li sembloit qu'elle avoit parlé trop asprement, elle leur disoit sa coulpe devant moy merveilleusement humblement, et mout s'accusoit, et 
recordoit les parolles qu'elle avoit dictes en agregeant sur $\operatorname{li}^{285}$. Mout me faisoit grand bien a l'ouïr, et puis m'en a faict bien la remembrance maintes fois. Je crois qui n'est nul pecheur en terre ${ }^{286}$ qui eust faict ${ }^{287}$ mout de pechez mortels, ce $^{288}$ il s'humilioit tant devant Dieu, et eust si grande repentance comme elle avoit quand elle avoit dict aucune chose ou elle se doubtoit que il eust peché, ou il n'y en ${ }^{289}$ avoit point si crioit a Dieu mercy, qu'il n'eust largement misericorde, tant doubtoit a courroucer Nostre Seigneur, et se gardoit de toutes occasions en soy et en autruy.

[28] Elle ${ }^{290}$ eut en sa fin de tres grandes maladies deux ans avant qu'elle trespassat ${ }^{291}$, lesquelles elle receut de son doux espoux tres doucement, et en grande patience les porta, et tres devotement sa vie fina en parfaite virginité et ${ }^{292}$ tres grande humilité et charité.

[29] Quand nostre tres reverente et saincte dame et mere vivoit, un des sergens monseigneur le roy Louys avoit un enfant qui cheoit de la grande maladie. Iceluy homme pria, en grandes larmes, a genoux et a main[s] jointes, devotement a la saincte dame qu'elle priast Dieu pour son enfant, qui estoit si cruellement malade, et elle s'inclina en signe qu'elle en prieroit Nostre Seigneur. Le pere s'en alla a son hostel et trouva que son enfant estoit gueri, et n'avoit plus celle maladie. Il retourna a madame, et s'agenouilla devant li et li dict: «Ma douce dame, vous souvient il de ce que je vous requis? Pour Dieu, dictes moy si vous en priastes Nostre Seigneur ». Elle li respondit: "Ouÿ». Lors il li dict: "Ma douce dame, je rends graces a Dieu et a vous que mon enfant est gueri, et je tiens fermement que c'est par vos prieres ». Et elle li dict : « Non, ne tenez pas que ce soit par moy, je ne suis pas telle que Dieu fasse ces choses pour ${ }^{293}$ moy ». Et il li disoit toujours qu'il tenoit que c'estoit par ses merites et par ses prieres. Quand elle vit qu'elle ne le pouvoit a ce mectre qu'il ne tenist que c'estoit par li, si li

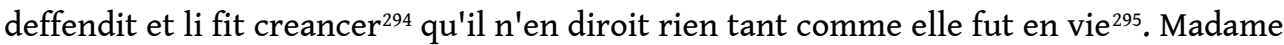
la grand reine Marguerite ${ }^{296}$ nous conta ceste chose, et nous ${ }^{297}$ dit que li hons ${ }^{298}$ qui estoit pere a l'enfant li conta ceste chose en verité.

[30] Encores quand madame vivoit, sœur Alis de Mucedent ${ }^{299}$ fut mout malade d'une fievre tierçaine. Elle eust devotion a madame, et li estoit advis que, si madame priast pour li, qu'elle fut guerie. Icelle seur Alis requit a seur Agnes d'Aneri ${ }^{300}$, qui adonc estoit abbesse, que elle y allast. Elle n'y osa aller pour la $^{301}$ reverence. Seur Alix en pria seur Agnes de Harecourt ${ }^{302}$, elle y alla, et li monstra la fiance que la malade y avoit. La saincte dame regarda seur Agnes de Harecourt, et sousrit mout amiablement, et tost aprés la malade fust toute gue-[p. 175]-rie de sa fiebvre. Je, sœur Agnes de Harecourt, qui portay la parole suis tesmoing de ceste chose, et aussi sœur Agnes d'Anery vit toutes ces choses.

87 [31] Sœur Sare de Houpelines ${ }^{303}$ eust une maladie moult perilleuse que l'on appelle l'orgueilleux ${ }^{304}$ : son corps estoit tout entrepris de boces et de taches, et cuidoit l'on que elle en ${ }^{305}$ deust mourir. Madame nostre saincte mere vint devers nous, et la regarda piteusement, et toucha la malade de ses benoistes mains, et tantost aprés la sœur fust toute guerie. De ceste chose plusieurs sœurs sont tesmoings, qui la virent malade et guerie.

[32] Frere Denys d'Estampes de l'ordre des freres mineurs, qui demeuroit en ceste abbaye pour administrer les sacremens aux sœurs, eut fiebvre quartaine par longtemps. Il fut present avec les autres freres mineurs quand on enhuila ${ }^{306}$ madame nostre saincte mere, et iceluy jour estoit le jour de sa fiebvre. Il fut gueri de sa fiebvre par les merites de la saincte dame, et onques puis n'eust fievre quartaine, et vesquit 
puis longtemps. Ceste chose il raconta a plusieurs sœurs et afferma estre vraye, et li convent le vit malade et gueri.

[33] Sœur Erembour de Cerceles ${ }^{307}$ dict en verité que ${ }^{308}$, icelle nuict que nostre benoiste mere trespassa, elle ouït devant matines une voix qui li dict : «In pace factus est locus ejus ». Et tantost icelle sœur Erembour alla a l'abbesse, et li dict que elle avoit ainsi ouÿ. L'on trouva que la saincte dame estoit trespassee, ou estoit au traict de la mort, et que c'estoit chose veritable de son trepas en icelle heure. Et semblablement en icelle heure sœur Jehane de Louvetaines ${ }^{309}$ ouït telle mesme voix.

90 [34] Sœur Clemence d'Argas ${ }^{310}$ dict en verité que la nuict que nostre saincte et reverente dame et mere trespassa, un peu devant matines, elle ouvrit la fenestre qui estoit pres son lict en intention pour sçavoir si elle orroit aucun en la court, car elle sçavoit bien que madame estoit pres de sa fin, et arregardoit l'air qui estoit tres bel et tres serain. Elle ouït une voix mout douce et mout melodieuse sur la maison ou elle gisoit, et l'ouït si longuement que ${ }^{311}$ li semble en verité que elle n'ouït onques si longue haleine en ceste mortelle vie. Icelle sœur Clemence mit son chef hors des fers de la

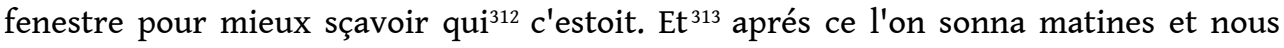
apporta l'on la nouvelle que madame nostre saincte mere estoit trespassee. Aussi sœur Aveline de Hennaut ${ }^{314}$ en celle heure ouït chants mout doux et mout melodieux, et se leva en son seant en son lict, mais elle ne sçait que ce fut. Nous croyons fermement que c'estoit la melodie des saincts anges qui conduisoient sa benoiste ame en la gloire du Ciel, car elle avoit loyaument honoré Dieu et servy en sa vie.

91 [35] Quand nostre saincte dame eut esté en terre par neuf jours, au neufviesme jour on la leva de la sepulture pour $1 a^{315}$ mettre en un autre cercueil plus convenable que celuy ou elle estoit. Elle ne sentit nulle mauvaise odeur, ains parut ainsi comme si elle dormit. Elle avoit les membres si beaux et si plains, et si traitables et si maniables, comme d'un tendre enfant, et la face li resplendissoit merveilleusement, si que toutes ces choses estoient merveilleuses a regarder. Et parce que on la demena tant, li yeux li ouvrirent liquels estoient si bels sans blesmir et sans müer qu'il ne sembloit pas que ils fussent estaints de mort. Nous la devestimes de la robbe que elle avoit eue neuf jours en terre, qui estoit si belle et si nette qu'il ne sembloit pas que elle eust oncques esté vestue. Pour ce que nous voulions avoir celle robbe comme reliques, nous la revestimes de nouvelle robbe, et la tretions tout ainsi comme nous voulions. Ce vit li convent et madame la contesse de Flandres Marguerite ${ }^{316}$, et madame Marie sa fille ${ }^{317}$ qui est nonnain, et la dame d'Audenarde ${ }^{318}$, et dame Huloys la veufve, et plusieurs autres personnes bourgeoises de Paris ${ }^{319}$, et monseigneur Guillaume de Guise ${ }^{320}$, chanoine de Vernon, qui fut son chapelain, et deux maçons avec ${ }^{321}$ qui estoient illec pour mectre le cercueil, et toutes ces personnes estoient dedans l'enclos.

92 [36] Par dehors, a la fenestre, furent tant de per-[p. 176]-sonnes qui la virent que nous ne sçaurions dire le nombre, et de religion et du siecle : entre lesquels furent frere Eude de Roni, maistre de divinité, qui fut son confesseur, frere Pierre de Ville ${ }^{322}$, frere Thomas du Plexi, frere Gilles de Salli, et plusieurs autres freres mineurs, et y estoit madame la fille au conte de Flandres, qui fut duchesse de Brabant ${ }^{323}$, et plusieurs autres dames et chevaliers, et bourgeois, et menu peuple. Nous ouvrismes la fenestre du monstier ${ }^{324}$ et levames le coffre et leur montrames la saincte dame comme un enfant en son berceau. Ils s'efforçoient qui mieux mieux de bailler leurs couvrechefs, leurs aniaux, leurs fermans, leurs chappeaux, leurs ceintures, leurs aumosnieres pour 
toucher au sainct corps par grand devotion, et ce qui y avoit touché, ils tenoient a reliques.

[37] Le frere Denys ${ }^{325}$, de qui nous avons dessus parlé, raconta de sa bouche que aprés huit jours que ceste nostre saincte dame et mere fut trespassee, il couvroit les autels de nostre eglise en Caresme, et une moult grande table qui estoit a l'autel monseigneur sainct Pierre cheut sur luy. Il estoit ${ }^{326}$ foible que de sa force il ne pouvoit de soy lever, et fut dessous le faiz par longue espace de temps. En ce peril et en celle mesaise il requit l'aide de nostre saincte dame, et tantost il s'eleva legerement de dessous ce grand faiz sans avoir nulle blessure, et fit son office vigoureusement, si comme devant ${ }^{327}$. Ceste chose il raconta a plusieurs sœurs qui en sont tesmoings.

[38] Frere Gilles de Salli, qui fut par long tems avec frere Eude de Roni, avoit un couvrechef que ceste saincte dame eut sur son chef en sa derniere maladie, et y sua la sueur de la mort. Il estoit malade de fiebvre tierçaine. Il mit par devotion de la saincte ce couvrechef sur son chef, et tantost il commença a suer, et fut gueri. Sœur Agnes d'Aneri, sœur Marie de Cambray ${ }^{328}$, sœur Marie de Tremblay ${ }^{329}$ ouïrent ceste chose de la bouche a ce frere Gilles, et en sont tesmoings.

[39] Sœur Ade de Rains ${ }^{330}$ dict en verité que une truye li emporta ${ }^{331}$ un des doigts de sa main, en telle maniere que elle n'eust point d'ongle en ce doigt par vingt ans et plus. Quand madame nostre reverente et saincte mere fut enterree, icelle sœur Ade prit de la terre entour le corps, et la lia sur ce doigt, et li tint par neuf jours. Au neufviesme jour elle le deslia. Il fut si tres purement gueri, que il n'y paroit qu'il y eust onques eu mal, et eut bel ongle et entier qui point $n$ 'en avoit devant, et ${ }^{332}$ sain toute sa vie. Li convent vit le doigt malade et sain.

[40] Sœur Ermesent de Paris ${ }^{333}$ demeura une fois toute seule au monstier ${ }^{334}$ sans congé quand li convent mangeoit au souper, en la nouvelleté que madame nostre benoiste mere fut trespassee. Une tres grande douleur la prit en son chef, et y sentoit avec trop grande ardeur. Et en cette douleur une grande peur la prit de ce qu'elle estoit demeuree sans congé, et pensa qu'elle iroit au refectoir avec les sœurs, et il ${ }^{335}$ li vint une grande volonté en son cœur, ainsi comme si ce fust une creature qui parlat a son cœur, et li dict : « Non feras, mais va a ta saincte dame, et li requiers aide ». Elle y alla, et se bouta dessoubs une fourme ${ }^{336}$ qui estoit sus le corps, et joint son chef et sa joue a la terre qui estoit dessus le corps, et la pria mout diligemment a grand efforcement et grandes larmes par longue piece, et aussi ${ }^{337}$ elle s'endormit illec. Quand elle se leva, elle se trouva toute guerie. Je, sœur Agnes de Harecourt, qui adonc estois en l'office d'abbesse, porte tesmoing de ceste chose, car icelle sœur Ermesent vint tantost a moy ainsi comme toute effrayee, et me dict que a peu qu'elle n'avoit perdu son sens de ${ }^{338}$ la douleur que elle avoit eue en son chef et de la peur qu'elle avoit eue, si Dieu et madame ne l'eussent guerie. Sœur Mahaut d'Escosse ${ }^{339}$, sœur Marie de Cambray et plusieurs autres sœurs portent tesmoings de ceste chose.

97 [41] Une autre de nos sœurs perdit son sens si outreement et fut si frenetique que quand elle pouvoit eschapper d'entre celles qui la gardoient, elle montoit sus les bancs et sus les huches. Et rompoit parfois ${ }^{340}$ pour prendre les araignees, et quand elle les ${ }^{341}$ en pouvoit prendre elle les mangeoit. Et se boutoit de-[p. 177]-ssoubs les tables, et queroit araignees et barbelotes ${ }^{342}$ esclotes $^{343}$, et partout ou elle les pouvoit trouver elle les mangeoit. Et mout d'autres ordures que nous ne voulons pas nommer elle mangeoit pour $^{344}$ la grande forcenerie ou elle estoit. Et en ceste maladie madame nostre benoiste mere, qui adonc vivoit, la visita mout humblement, et en avoit mout grande 
compassion. Et ceste maladie dura a celle sœur trois mois et demy puis que nostre saincte dame fut trespassee. On l'emena une nuict a la tumbe de la saincte, et y veilla toute la nuict, et les sœurs avec li qui furent en oraison, et prioient madame pour li qu'elle la vousit saner ${ }^{345}$ de cette maladie. Tout ainsi comme la nuict s'en alla son sens li revint, et a la journee elle eut son sens si apertement comme elle avoit oncques eu, et oncques puis ne cheut en celle maladie. Li convent vit ceste chose, et en est tesmoing.

[42] Sœur Julienne ${ }^{346}$ dict en verité que elle estoit en grande ${ }^{347}$ chaleur de fiebvre, et en celle chaleur elle eut tres grand desir de boire par devotion au hanap ou nostre saincte dame beuvoit en sa vie ${ }^{348}$. Si tost comme elle y eut beu, elle fut alegee de la chaleur de la fiebvre, et fust assez tost toute guerie, et plus de dix ans aprés elle ne sentit fiebvre.

[43] Icelle mesme sœur Julienne avoit un livre, lequel elle aymoit mout pour la devotion de ce qu'il avoit esté $\mathrm{a}^{349}$ nostre saincte dame. Iceluy livre fust perdu par male garde, de quoy elle fut mout mesaisiee. Elle alla a sa tumbe, et li requit mout a certes en pleurant que elle li rendit, car elle l'aimoit mieux parce qu'il avoit esté sien. Nostre douce saincte mere li apparut en dormant, et li dict que le livre estoit perdu et qu'elle en requit monseigneur le roy sainct Louys son frere. Quand la sœur s'esveilla, elle fist l'oraison au sainct et promit a ambes deux ${ }^{350}$ au sainct ${ }^{351}$ une livre de cire par le congé de l'abbesse. Et tantost comme on alla querre le livre, on le trouva; et par plusieurs jours devant ce on ne le pouvoit trouver, et si l'avoit l'on mout quis.

[44] Sœur Ermengart de Chartres ${ }^{352}$ avoit une mout fort fiebvre tierçaine: si eut volonté et devotion de faire une chandelle de son long ${ }^{353}$ a madame, et la requit. Elle fut guerie mout nettement de sa fiebvre, si que oncques puis n'en eut point. Elle alla a la tumbe et fit l'offrande le plus tost qu'elle peut.

101 [45] Madame la grand reine Marguerite, mere au roy de France ${ }^{354}$, fit apporter monseigneur Philippe ${ }^{355}$, le fils au roy, qui fiebvre avoit, en esperance qu'il fut gueri. Elle le fit coucher enpres la tumbe nostre saincte dame sa reverente tente. Il fut gueri, si comme il mesme a puis dict devant plusieurs sœurs que elle le guerit, et dict que li $\mathrm{en}^{356}$ souvient bien.

102 [46] Sœur Marguerite de Guyse ${ }^{357}$ avoit une buchete en l'un de ses yeux. Elle estoit a telle angoisse que elle ne pouvoit ouvrir l'œil. Elle requit madame que elle li aidast, et mit sur son œil des vestemens de la benoiste saincte, et tantost elle fut guerie.

103 [47] Sœur Marie de Cambray avoit si perdue l'ouïe que elle n'oyoit ainsi comme nulle goute, et ne sçavoit respondre a ce que l'on li disoit, si qu'elle en pleuroit forment souvent et en estoit mout mesaisiee. Elle eut devotion de requerre nostre saincte dame, et fut en oraison a sa tumbe par neuf jours, et de jour en jour elle amendoit, et au neufviesme jour elle fut toute guerie.

[48] Sœur Isabel de Crecy ${ }^{358}$ dict en verité qu'elle estoit mout grefvement malade et en peril de mort d'une enfleure qui la tenoit entour les flancs si forment qu'elle ne se pouvoit dresser. [Nulle] chose que l'on li fist ne la pouvoit alleger. Les sœurs li apporterent l'oreiller qui avoit esté en la sepulture madame par neuf jours. Tantost comme elle le mit sur la fourcele ${ }^{359}$, elle allegea et fut guerie de la maladie. Sœur Agnes de Harecourt, sœur Agnes d'Anery, sœur Marguerite de Guyse et plusieurs autres sœurs se recordent bien de ceste chose.

[49] Une autres fois icelle mesme sœur Isabel avoit trop grande douleur a la fourcele, et sœur Ade de Rains qui adonc vivoit, que madame avoit guerie de son doigt, li dit: "Allez a la tumbe madame, et prenez de la terre qui est sus la tumbe, et en me-[p. 178]- 
tez sur vostre fourcele, et vous serez toute guerie ». Icelle sœur Isabel dict en verité que, en l'heure qu'elle mit de celle terre sur la fourcele, elle fut toute guerie.

[50] Sœur Erembour de Cerceles dict en verité que elle estoit trop griefvement malade, et li tenoit celle douleur dessous la mammelle si que elle ne pouvoit avoir l'halaine. Elle eut fiance en nostre saincte benoiste dame et mere et la requit. Et aucune des choses qui avoient esté a la saincte dame elle mit au lieu ou malade estoit, et tantost elle allegea et fut guerie. Plusieurs sœurs virent et asseurent ceste chose.

[51] Sœur Alis de Mucedent avoit la bouche torte ${ }^{360}$, et l'œil, et la face, et le nez, ainsi comme de paralysie, et la parole li estoit si empeschee que a peine la pouvoit l'on entendre. Et en cet estat elle fut bien trois sepmaines ou un mois ${ }^{361}$ : nulle chose que l'on li pouvoit faire de physique ne li pouvoit rien valoir. Et adoncques il li vint devotion et volonté que elle prit des choses que elle avoit qui furent a nostre saincte dame et mere, et que elle les portast a son col, et que elle la requit et allast a sa tumbe. Elle y alla par huict jours faire l'oraison, et a l'huictiesme jour elle offrit une chandele de la grossesse de son chef et de la longueur de son visage. Et tantost aprés ce elle fut toute guerie, et onques puis n'en fut malade, si comme il appert. Et de ceste chose sœur Agnes d'Aneri, qui la gardoit, en porte tesmoing, et mout d'autres sœurs qui la virent ${ }^{362}$ toute guerie.

[52] Sœur Marie de Tremblay dict en verité que elle estoit allee esbatre vers le vivier qui est en nostre maison, et s'assit sus les quarreaux ${ }^{363}$ qui sont dessus le vivier, et y fut une bonne piece pour prendre de l'air, car elle estoit mout lassee des offices qu'elle avoit eu a faire. Et si comme elle estoit illec, le quarreau sur quoy elle se debat ${ }^{364}$ despeça ${ }^{365}$ dessous li, et cheut au vivier, et brisa la glace, et la sœur cheut avec au ${ }^{366}$ vivier, et coula dedans le vivier jusques outre la ceinture, et couloit jusques au fonds. Et il li remembra de nostre saincte dame, et ${ }^{367}$ elle la requit mout de cœur, et dict : « Ma douce dame, sauvez moy, si vrayement comme je suis vostre fille », et tantost Nostre Seigneur la delivra merveilleusement, si comme elle qui estoit en grand peril de mort. Et tantost elle s'en issit legerement de l'eau, et dict bien que elle n'eut oncques si grand angoisse ne si grand peur de mort, et proposa en son cœur de mieux faire. Plusieurs sœurs virent la griefveté qu'elle avoit quand elle fut issue de ce grand peril. Ceste chose elle recorda a plusieurs sœurs, et trouva l'on le quarrel despecié, si comme elle avoit dict.

109 [53] Icelle mesme sœur Marie de Tremblay gardoit seur Desiree ${ }^{368}$, malade, que l'on luy avoit baillee a garder. La malade li dict que elle li allast querre de l'eau de la fontaine du vivier, et sœur Marie li dict que elle avoit trop grand peur et trop grand horreur, pour ce qu'il estoit nuict aussi comme au premier somme. Et toute preste ${ }^{369}$ pour accomplir la volonté de la malade, elle prit une chandele et un pot, et y alla. Si comme elle y alloit, l'Ennemy vint encontre li en semblance d'un chien vert, et avoit les yeux rouges et estincelans, et si grands et si gros, qu'il sembloit que ce fussent yeux de vaches ${ }^{370}$. Elle avoit si grand peur qu'il li sembloit que tout son corps fust esmeu, et que l'on li tirast les cheveux amont. Et tousjours il venoit encontre son visage, et la destourba ${ }^{371} \mathrm{li}^{372}$ d'aller, que elle ne peust oncques aller jusques a l'eau, ains la convint retourner. Et au retourner elle se seigna, et le bouta de son bras arrieres, et dit: «Pater, in manus tuas commendo spiritum meum ${ }^{373}$, et en celle heure il se departit de li si que elle ne sceut qu'il devint. Elle prit son tour a aller a la fontaine de la lavanderie, et quand elle fut illec a la fontaine, il se mit outre ${ }^{374}$ li et le fourvel ${ }^{375}$, et li saillit sur les espaules, et la vouloit estrangler. Ainsi comme elle s'en retourna pour aller s'en, elle se seigna, et dit : 
«A, ma douce dame, deffendez moy de ce diable si comme je suis vostre fille, et je promets a Dieu, et a Nostre Dame, et a vous, que je me confesseray generaument et amenderay ma vie ». Et ainsi comme elle vouloit entrer en la maison ou la malade gisoit, elle [p. 179] cheut ainsi comme toute pasmee, et n'eut onques pouvoir de fermer l'huis, et li pot que elle tenoit en sa main cheut et fut brisé. La malade, qui ne s'en pouvoit remuer, ouït bien les cris que seur Marie cryoit, et li disoit: "Signez vous, signez vous». Sœur Desiree fut tesmoing de ceste chose, se elle fut en vie. Sœur Jehanne de Louvetaines, qui garda grand piece la malade, et sœur Julienne tesmoignent que seur Desiree leur dict plusieurs fois ceste chose en sa vie.

110 [54] $]^{376}$ Sœur Jehanne de Louvetaines dit en verité que en une grande maladie que elle eut, qui li dura trois mois, elle se voua a madame nostre saincte mere, et li pria mout de cœur que elle priast Nostre Seigneur qu'il la sanast, et disoit ainsi : « Ma douce dame, ma douce mere, je vous prie que vous me donniez sancté, car je croy certainement que vos merites sont plus grands que la necessité que j'ay», et ainsi prioit en grandes larmes et plusieurs fois. Et li avint qu'une nuict elle fut mout griefvement malade, en telle maniere que il li sembloit que elle ne peust durer, et appella sœur Mahaut d'Escosse qui la gardoit, et li dict : «Signez moy, et me recommandez a madame nostre benoiste mere", et tantost s'endormit. En ce dormir il li sembloit que elle voyoit madame, et s'agenouilloit devant li, et li faisoit sa priere ainsi comme devant a jointes mains, et madame li respondoit: «Allez a mon frere». Apres il li sembloit que elle voyoit mout de gens ainsi comme pelerins aller a la tumbe monseigneur le roy, et li estoit advis que elle n'y pouvoit aller, pour ce si crioit au roy: «Sire, je crie a vous misericorde, senez ${ }^{377}$ moy ». Et li sembla que elle fut portee a la tumbe monseigneur le roy, et que madame y estoit. Et li sembloit que li roy tenoit sa main dextre en haut dessus la tumbe, et madame li disoit: «Sire, segnez ou sanez ceste sœur». Et il la segna ${ }^{378}$, et li dict: "Vous serez guerie dedans huict jours ». Et tantost comme elle fut esveillee, elle conta ceste chose a seur Mahaut qui la gardoit, et li dit : " Je suis guerie». Et cet verité que el fut tantost guerie : li convent la vit malade, et vit la santé.

111 [55] ${ }^{379}$ Icelle mesme sœur Jehanne de Louvetaines eut une mout griefve maladie, qui li dura bien trois ans, et peu avoit d'esperance de jamais avoir santé pour la griefveté de sa maladie. Elle se voua a madame nostre saincte mere, et li promit que elle jeusneroit en pain et en eau par trois samedis. Quand elle eut ainsi jeusné, si dict a nostre sainte dame : «A, ma douce dame, or ay je jeusné par trois samedis en pain et en eau qui mout m'ont cousté, et encore ne suis je point confortee ». Elle s'endormit et li sembla que elle fust portee sur la tumbe madame, et que madame se seoit sur la tumbe, dont la malade fut un peu espouventee. Et li souvint, et dict a soy mesmes: "C'est celle a qui tu requiers aide ». Et sembloit a la sœur que madame venoit encontre li, et elle disoit a madame: "Madame, je vous prie que vous m'aidez envers Nostre Seigneur et me sanez », et madame la prit entre ses mains, et li dit: "Allez a mon frere ». Adonc il sembloit a la sœur que elle voyoit une procession de roys mout noblement appareillez et tous couronnez, et en la fin de celle procession estoit monseigneur le roy Louys. Madame prit la sœur et la mit devant luy, et li dit qu'il la segnat. Monseigneur le roy segnat la sœur, et li dict : « Vous serez toute guerie ». Et certainement la sœur fut toute guerie, si comme il apparut aprés que toutes virent que elle fut guerie, et oncques puis n'eut tache de la maladie.

112 [56] Il avint a sœur Sare de Houpelines que un mout felon chien de nostre maison, qui mout avoit faict des maux aux sœurs, eschappa, et li va saillir au visage, et elle mit sa 
main au devant. Le chien la prit par la main, et li fist douzes playes en la main et au bras. Aprés il la prit par la cuisse, les le genouil, et li fit mout de grandes playes. Illec avoit mout de sœurs qui s'efforçoient de li secourre, mais elles ne pouvoient oster le chien de li. Adonc sœur Sar requit Nostre Seigneur, et Nostre Dame, et nostre saincte mere, madame Isabelle, a qui elle dit ainsi : « Ma douce mere, me laisserez vous manger aux chiens?». Et tantost le chien s'en alla de sa volonté et la laissa, et elle demeura mout griefvement [p.180] navree. Aprés ce la cuisse de la sœur enfla et aggreva si forment que l'on cuida que elle deut mourir. Et adonc elle demeura par congé toute seule a la tumbe madame tant comme li convent mangea, et pria Dieu, et Nostre Dame, et madame nostre saincte mere, que elle ly aidast. Et tantost avant que li convent eust mangé, elle se sentit allegee de sa grande maladie ${ }^{380}$ de l'enfleure, et est toute guerie. Et ce vit sœur Isabel de Tremblay qui la gardoit et plusieurs autres sœurs, et nous le voyons que elle est toute guerie.

113 [57] Plusieurs sœurs ont veü grand clarté plusieurs fois entour la tumbe nostre saincte dame et mere entour l'heur de matines, et autres choses devotes qui longues sont a raconter.

114 [58] Li breviere sœur Agnes de Paris ${ }^{381}$ cheut en eau tout ouvert, et fut si du tout mouillé dedans et dehors qu'il ne sembloit pas qu'il fust jamais convenable a lire la lettre. L'on le porta par devotion sus la tumbe a nostre saincte dame, et le laissa l'on illec entour trois heurs. Il fut restauré en son premier estat, et est beau et lisable comme devant ce qu'il cheut en l'eau ${ }^{382}$.

115 [59] Icelle mesme sœur Agnes avoit si mal dedans le conduit de sa gorge que elle ${ }^{383}$ estoit mout effrayee. Si tost comme elle eut mis sur le mal aucunes des choses qui avoient touché au sainct corps de madame, elle rendit par la bouche ainsi comme palu ${ }^{384}$, et fut nettement guerie.

116 [60] Nous ${ }^{385}$ pourrions raconter a briefves paroles les biens et les consolations spiritueles que elle a faict aux personnes qui devotement li ont requis aide de quelconque tribulation et mesaise. L'on la requiert: elle secourt et conforte isnelement ${ }^{386}$ qui de vray cœur la prie.

117 [61] Une femme de Paris, qui a nom Agnes la Coffriere, avoit un enfant mout griefvement malade, et n'i attendoit l'on que la mort. Elle l'aimoit mout car elle n'avoit plus d'enfans. Elle et autres personnes avoient veillé devant l'enfant, pour ce que l'on attendoit sa fin. L'on la fit aller reposer, elle s'endormit, et en ce dormir il li sembla que elle ouyt une voix qui li dict : « Agnes, voue ton enfant a madame Isabelle pres de Saint $\mathrm{Clou}^{387}$, et li offre le hanap que ton pere te donna, et ton enfant sera gueri ». Lendemain elle vint a nostre maison en pelerinage, et offrit li hanap, et li enfant fut gueri.

[62] Une femme de Surenes ${ }^{388}$, qui a nom Agnes, perdit la veue de ses yeux par force de maladie. Elle se fit amener a nostre abbaye et se voua a nostre saincte dame, et li promit deux yeux de cire. Si tost comme elle eut fait son vœu de l'oraison au monstier ${ }^{389}$, elle vit, et en ce jour elle receut plainement la veue.

[63] Une pucelle qui estoit deux lieues loing de nostre eglise estoit en peril de perdre sa virginité. Et la nuit avant que elle fut livree, nostre saincte dame li apparut en ${ }^{390}$ dormant, et li dit: "Leve sus, va a m'abbaye qui est pres de Sainct Clou, et tu seras delivree ». La pucelle se leva tres matin, et comme elle ne sceut quelle part l'abbaye fut, elle accourut tout droit, et vint si suant et si lassee de courre que a peine pouvoit elle avoir s'haleine. Et pour le grand desir qu'elle avoit d'estre sauvee, elle laissa son sercot 
au boias ${ }^{391}$ pour plus tost accourre, et fut $\mathrm{li}^{392}$ sercot trouvé. Si comme Dieu veut, et d'illec en avant la pucelle demeura en sa neteté, et mena belle vie et honneste, si comme tesmoingnent les personnes entre qui elle demeura.

[64] Deux hommes devers Tournay ${ }^{393}$ vindrent a nostre abbaye, et apporterent a l'offrande deux chandeles de leur long. Et requirent que l'on leur montrast la tumbe nostre saincte dame, et dirent que ils estoient en prison et en peril de la mort de la corde ${ }^{394}$, et une voix leur dict : «Vouez vous a madame Isabel pres de Saint Clou, et vous serez delivrez ». Et pour ce ils estoient venus, et requeroient a grande instance a voir la tumbe de la benoiste dame. On leur respondit qu'il n'estoit pas accoustumé d'ouvrir souvent la fenestre : on fit ardre leurs chandeles entour la tombe, et ils s'en ralerent tous delivrez.

[65] La guete ${ }^{395}$ de nostre maison nettoyoit le monstier ${ }^{396}$, et estoit ${ }^{397}$ aux voutes en [p. 181] une corbeille tiree a cordes par engin. La corde rompit, et il cheut sur les estaux du monstier ${ }^{398}$, et fut mout cassé, et eut une playe en son chef de ce qui se bleça au choir, et fut merveille qu'il ne fut tout ecervellez, et doubta l'on qu'il ne mourut, et convint les freres venir a grand haste pour luy confesser. Les sœurs en eurent mout grand pitié, et le vouerent a madame nostre saincte mere, et dedans brief temps il fut tout gueri, et n'eut nul mehaing ${ }^{399}$ de la blessure.

[66] Quand madame la reine ${ }^{400}$ demeuroit en nostre maison, li valet a son aumonier ${ }^{401}$ fut malade, et cheut en forte frenaisie. Bonnes gens eurent pitié de luy, et le vouerent a madame nostre saincte dame et mere, et li offrirent une chandelle du long au malade. Tantost li malade revint en son sens, et fut gueri de la frenaisie, et se confessa, et s'appareilla. Et ce virent le frere de nostre maison et plusieurs autres gens.

[67] Philippe, procureur de nostre abbaye, avoit fiebvre tierçaine si aspre ${ }^{402}$ que l'en doubtoit qu'il ne perdit son sens. Il ne pouvoit suer pour rien que l'en li fist. Si tost comme l'on le coucha sus l'oreiller que madame nostre saincte mere eut en soubs son chef, tantost il sua et fut tout gueri.

[68] Le fils Richart ${ }^{403}$, aprés ce qu'il eust eu sus soy de la terre qui fut prise entour la sepulture de la saincte dame, fut gueri de fiebvre quotidiane que il avoit eu grand piece.

[69] XL miracles.

\section{NOTES}

1. Guillaume de Saint-Pathus, Vie de saint Louis, H.-F. Delaborde éd., Paris, 1899, p. 132.

2. Jean de Joinville, Vie de saint Louis, J. Monfrin éd., Paris, 1995, p. 344 : « L'abbaïe des Cordelieres de Saint Clou, que sa seur ma dame Ysabiau fonda par son otroi ».

3. Ch. Du Fresne, sieur Du Cange, Histoire de saint Louys IX roy de France écrite par Jean sire de Joinville sénéchal de Champagne, Paris, 1668, p. 169-181.

4. À l'exception d'un travail très recent: S. L. FIELD, The Princess, the Abbess, and the Friars : Isabelle of France (1225-1270) and the Course of Thirteenth-Century Religion History, Ph.D., Northwestern University, Evanston, Illinois, 2002. 
5. H. L. Bouquet, L'ancien collège d'Harcourt et le lycée Saint-Louis, Paris, 1891, p. 46-56.

6. G. MLYNARCZYK, Ein Franziskannerinnenkloster im 15. Jahrhundert. Edition und Analyse von Besitzinventaren aus der Abtei Longchamp, Bonn, 1987, p. 57.

7. Charles I ${ }^{\mathrm{er}} \mathrm{d}^{\prime}$ Anjou, né en 1227, est le dixième fils de Louis VIII et Blanche de Castille. Il est comte de Provence, roi de Naples et de Sicile. Il meurt en 1285.

8. $\S 40$ de notre édition de texte, infra, p. 91.

9. L. Carolus-Barré, Le procès de canonisation de saint Louis (1272-1297). Essai de reconstitution, Rome, 1994, p. 20-23.

10. Déposition de Charles d'Anjou pour la canonisation de saint Louis reconstituée par L. Carolus-Barré, Le procès de canonisation de saint Louis (1272-1297), op. cit., p. 68.

11. G. Klaniczay, «The Cult of Dynastic Saints in Central Europe : Fourteenth-Century Angevins and Luxemburgs ", dans The Uses of Supernatural Power, Princeton, 1990, p. 111-128 ; les saints sont la caution de la légitimité d'une dynastie : cf. A. Vauchez, "Beata stirps : sainteté et lignage en Occident aux xiii ${ }^{\mathrm{e}}$ et xiv ${ }^{\mathrm{e}}$ siècles ", dans G. Duby et J. Le Goff dir., Famille et parenté dans l'Occident médiéval, Rome, 1977, p. 397-406. Louis IX avait déjà essayé de faire reconnaître son frère Robert d'Artois comme martyr pour être mort à la croisade ; cf. J. Le Goff, Saint Louis, Paris, 1996, p. 267.

12. $\S 3$ de l'édition du texte.

13. Ibidem.

14. Le titre « roi d'Espagne » est vraisemblablement une modification de l'éditeur du $\mathrm{xvii}^{\mathrm{e}}$ siècle. Au xiii ${ }^{\mathrm{e}}$ siècle, Alphonse VIII aurait été nommé roi de Castille ou imperator Hispanie. Cf. J. M. Jover Zamora dir., Historia de España, IX, Madrid, 1998, p. 27.

15. J. de Laborde éd., Layettes du trésor des chartes, III, p. 246-247, nº 4184 et p. 256-257, $n^{\circ} 4194$; A. Lewis, Le sang royal. La famille capétienne et l'État, France, $X^{e}-X_{I V}{ }^{e}$ siècles, Paris, 1986, p. 232 et n. 128, p. 371.

16. $\S 3$ de l'édition du texte.

17. $\S 1$ de l'édition du texte.

18. $\S 4$ de l'édition du texte; Matt. 11, 29 : « Chargez-vous de mon joug et mettez-vous à mon école »; c'est le mode de vie monastique d'Isabelle qui est ici mis en valeur, selon l'idée de Bernard de Clairvaux : la vie monastique reproduit la vie des apôtres, école du Christ ; cf. J. Verger et J. Jolivet, Bernard-Abélard ou le cloître et l'école, Paris, 1982, p. 112. 19. Sur cette fonction d'exemplarité des princesses et sur le thème de la « vraie noblesse » : cf. J.-P. Boyer, « Vertus privées et bien public. Reines et princesses selon la prédication De mortuis à la cour de Naples », dans Reines et princesses au Moyen Âge, Cahiers du CRISIMA n ${ }^{\circ}$ 5, I, Montpellier, 2001, p. 420-421.

20. $§ 11$ de l'édition du texte. L'insistance d'Agnès d'Harcourt sur la véracité de ses dires peut être comparée à celle, plus tardive, de Jean de Joinville. Sur l'importance du témoignage oral pour l'historien médiéval, cf. B. Guenée, Histoire et culture historique dans l'Occident médiéval, Paris, 1980, p. 78.

21. $§ 7$ de l'édition du texte.

22. B. Guenée, Histoire et culture historique dans l'Occident médiéval, op. cit., p. 98.

23. Claire est morte le 11 août 1253 à Assise, après avoir vécu plus de quarante ans au monastère de Saint-Damien. Sa canonisation eut lieu en 1255. Elle donne lieu à la rédaction de deux vitae. La plus largement utilisée est la vie en prose, appelée Legenda sanctae Clarae virginis, rédigée par Thomas de Celano en 1256. Cf. M. Bartoli, Claire d'Assise, Paris, 1993, p. 19-20. Le monastère de Longchamp possède au xiv ${ }^{\mathrm{e}}$ siècle une traduction fragmentaire de cette vie en français, actuellement conservée aux Archives nationales, LL 1601. Cf. F. Berriot, « Les manuscrits de l'abbaye de Longchamp aux 
Archives de France et la Vie de sainte Claire inédite (début xive) » Archivum Franciscanum Historicum, 79, 1986, p. 329-358.

24. Née en 1207, elle est la fille d'André II de Hongrie. Mariée en 1221, elle devient veuve à vingt ans et meurt à vingt-quatre. $\mathrm{Cf}$. M. Goodich, « Une enfant sainte, une sainte des enfants : l'enfance de sainte Élisabeth de Hongrie (1207-1231)», dans E. Becchi et D. Julia dir., Histoire de l'enfance en Occident, de l'Antiquité au XVII siècle, I, Paris, 1996, p. 134-139.

25. Ouvrage composé en 1236 par Ulrich von Durne, qui servit de source aux hagiographies postérieures.

26. M. Goodich, "Ancilla dei : the servant as saint in the late Middle Ages ", dans J. KIRSHNER et S. F. WEMPLE éd., Women in the medieval world, Oxford, 1985, p. 119-136.

27. Jean de Joinville, Vie de saint Louis, op. cit., p. 48.

28. Geoffroy de Beaulieu, Vita et sancta conversatio piae memoriae Ludovici quondam regis Francorum, dans Recueil des historiens des Gaules et de la France, F. Daunou et J. Naudet éd., XX, Paris, 1840, p. 3-27 ; L. Carolus-Barré trad. et éd. (extraits), Le procès de canonisation de saint Louis, op. cit., p. 29-59.

29. Guillaume de Chartres est un Dominicain, chapelain de Louis IX durant la première croisade. Il a partagé sa captivité. Guillaume de Chartres, De Vita et actibus inclytae recordationis regis Francorum Ludovici et de miraculis quae ad ejus sanctitatis declarationem contingerunt, dans Recueil des historiens des Gaules et de la France, XX, op. cit., p. 27-41. 30. G. MLYNARCZYK, Ein Franziskannerinnenkloster im 15. Jahrhundert. Edition und Analyse von Besitzinventaren aus der Abtei Longchamp, op. cit., p. 35, 54, 57, 189.

31. $\S 19$ de l'édition du texte.

32. $\S 24$ de l'édition du texte.

33. Sur les prises de parole féminines, voir V. Jouët, « Mots, occasions et contenus de la parole des reines et des princesses dans la Chronique du religieux de Saint-Denis ", dans Reines et princesses au Moyen Âge, op. cit., p. 449-467.

34. Le chancelier de Paris Aymeric de Veire a également été confesseur d'Isabelle, mais il meurt vers 1262 ; P. Glorieux, Répertoire des maîtres en théologie de Paris au XIII ${ }^{e}$ siècle, II, Paris, 1933, p. 53 et I, p. 332.

35. $§ 26$ de l'édition du texte.

36. Cf. J. Dalarun, « Hors des sentiers battus. Saintes femmes d'Italie aux xiii ${ }^{\mathrm{e}}-\mathrm{xiv}^{\mathrm{e}}$ siècles ", dans Femmes. Mariages. Lignages. Mélanges offerts à Georges Duby, Bruxelles, 1992, p. 102.

37. Isabelle est très obéissante face à son confesseur : $§ 14$ et 26 de l'édition de texte. 38. Tel Ph. de Dabra Bizen, l'enfant-saint peut déclarer que « Mon père et ma mère sont le Seigneur. "; D. Lett, L'enfant des miracles. Enfance et société au Moyen Âge (XII ${ }^{e}$-XIII ${ }^{e}$ siècles), Paris, 1997, p. 221.

39. A. Vauchez, La sainteté en Occident aux derniers siècles du Moyen Âge, d'après les procès de canonisation et les documents hagiographiques, Rome, 1988, p. 499.

40. $§ 34$ de l'édition du texte.

41. $\S 35$ de l'édition du texte.

42. Thomas de Celano, Vita prima, T. Desbonnets et D. Vorreux trad., Paris, 1968, p. 314.

43. A. Vauchez, La sainteté en Occident aux derniers siècles du Moyen Âge, op. cit., p. 499-507 ; J.-P. Albert, Odeurs de sainteté. La mythologie chrétienne des aromates, Paris, 1990.

44. $§ 35-36$ de l'édition du texte. 
45. A. Vauchez, « Le miracle entre la vie quotidienne et l'utilisation pastorale », dans Saints, prophètes et visionnaires. Le pouvoir surnaturel au Moyen Âge, Paris, 1999, p. 39-55. 46. Thomas de Celano, Sainte Claire d'Assise, sa vie et ses miracles, M. Havard de la Montagne trad., Paris, 1917.

47. S. Chennaf et O. Redon, «Les miracles de saint Louis ", dans J. Gelis et O. Redon dir., Les miracles miroirs des corps, Saint-Denis, 1983, p. 53-85.

48. $\S 40,41$ et 48 de l'édition du texte.

49. Guillaume de Saint-Pathus est un Cordelier, confesseur de Marguerite de Provence puis de sa fille Blanche de France. À sa demande, il rédige vers 1302-1303 une Vie de saint Louis et les miracles du roi d'après le dossier du procès de canonisation ; Guillaume de Saint-Pathus, Miracles de saint Louis, P. B. Fay éd., Paris, 1931.

50. S. Chennaf et O. Redon, «Les miracles de saint Louis », loc. cit., p. 78.

51. A. Vauchez, La sainteté en Occident aux derniers siècles du Moyen Âge, op. cit., p. 410-434.

52. $\S 12$ de l'édition du texte.

53. C. W. Bynum, Jeûnes et festins sacrés. Les femmes et la nourriture dans la spiritualité médiévale, trad. fr., Paris, 1994.

54. § 15 de l'édition du texte.

55. A. Vauchez, La sainteté en Occident aux derniers siècles du Moyen Âge, op. cit., p. 406.

56. Le sang au Moyen Âge, Les cahiers du CRISIMA n ${ }^{\circ}$ 4, 1999 ; J.-P. Albert, Le sang et le Ciel. Les saintes mystiques dans le monde chrétien, Paris, 1997, p. 200-212.

57. G. Dickson, « Flagellants », dans A. Vauchez dir., Dictionnaire encyclopédique du Moyen Âge, Paris, 1997, p. 601-602.

58. $\S 15$ de l'édition du texte.

59. $\S 7$ et 28 de l'édition du texte.

60. $\S 63$ de l'édition du texte.

61. C. W. Bynum, Jeûnes et festins sacrés, op. cit., p. 178.

62. A. Vauchez, La spiritualité du Moyen Âge occidental, Paris, 1970, p. 162.

63. Thomas de Celano, Sainte Claire d'Assise, sa vie et ses miracles, op. cit., p. 17 et 69.

64. R. Folz, Les saintes reines du Moyen Âge en Occident ( $\mathrm{VI}^{e}$-XIII ${ }^{e}$ siècles), Bruxelles, 1992, p. 81.

65. J. Le Goff, Saint Louis, op. cit., p. 150.

66. Le projet de mariage faisait peut-être partie des initiatives de réconciliation de Louis IX avec Frédéric II : cf. J. Le Goff, Saint Louis, op. cit., p. 117 ; W. C. Jordan, Louis IX and the Challenge of the Crusade. A Study in Rulership, Princeton, 1979, p. 9.

67. $\S 20$ de l'édition du texte.

68. AN J $403 \mathrm{n}^{\text {os }} 2$ et $2^{\text {bis }}$, testament de Louis VIII, juin 1225. A. Teulet éd., Layettes $d u$ trésor des chartes, II, Paris, 1866, p. 54-55, nº 1710 : « Item donamus et legamus Elisabet karissima filie nostre viginti milia librarum ».

69. $\S 7$ de l'édition du texte.

70. ID. et en note.

71. Thomas de Celano, Sainte Claire d'Assise, sa vie et ses miracles, op. cit., p. 17-18.

72. M. Goodich, « Une enfant sainte, une sainte des enfants : l'enfance de sainte Élisabeth de Hongrie (1207-1231) », loc. cit., p. 134-139.

73. M. CATHERINE, Margaret : Princess of Hungary, Oxford, 1945.

74. C. DAUPHIN et alii, « Women's Culture and Women's Power : Issues in French Women's History », dans K. OfFEN, R. ROACH PIERSON et J. RENDALl dir., Writing Women's History. International Perspectives, Indianapolis, 1991, p. 107-127. 
75. $§ 5$ de l'édition du texte.

76. $\S 13$ de l'édition du texte.

77. Claire d'Assise (1194-1253) rédige sa règle de 1247 à 1253, première règle écrite par une femme et pour des femmes. Cf. M. P. Alberzoni, Chiara e il papato, Milan, 1995.

78. $\S 23$ de l'édition du texte.

79. Bulle d'Alexandre IV Sol ille verus du 2 février 1259 ; J. H. Sbaraleae, Bullarium franciscanum, III, Rome, 1765, p. 64. Bulle d'Urbain IV Religionis augmentum, 27 juillet 1263 ; J. H. Sbaraleae, Bullarium franciscanum, op. cit., II, 1761, p. 477.

80. P. Gilli, « Un saint lettré : la vie du bienheureux Ambroise », dans P. Gilli dir., Former, enseigner, éduquer dans l'Occident médiéval (110-1450), I, Paris, 1999, p. 165-166.

81. Thomas d'Aquin a été canonisé en 1323 surtout pour son œuvre théologique.

A. Vauchez, La sainteté en Occident aux derniers siècles du Moyen Âge, op. cit., p. 455.

82. $\S 43$ de l'édition du texte.

83. $\S 58$ de l'édition du texte.

84. On pouvait voir aux pieds du gisant d'Isabelle à Longchamp deux petites religieuses, dont l'une tenait un livre. Cf. J. Pien, G. CuYPers et J. Stiltingh, Acta sanctorum, Augusti, VI, Anvers, 1743, p. 791.

85. Cambridge, Fitzwilliam Museum 300 ; Cf. S. C. COCKERELL, A Psalter and Hours Executed before 1270 for a Lady Connected with Saint Louis, probably his Sister Isabelle of France, Londres, 1905.

86. Exemple : AN L $1026 \mathrm{n}^{\circ} 2$ : inventaire des livres lors de l'élection d'Agnès d'Harcourt.

87. A. Vauchez, Les laïcs au Moyen Âge. Pratiques et expériences religieuses, Paris, 1987, p. 111 ; J. Dalarun, « Hors des sentiers battus... », dans Femmes, mariages, lignages, op. cit., p. 79-102.

88. $\S 18$ de l'édition du texte ; elle est la sœur de Jean de Montfort, compagnon de saint Louis lors de sa première croisade. Cf. M. J. L'Hermitte, Précis sur la ville de MontfortL'Amaury, et histoire chronologique des seigneurs de cette ville, Paris, 1825, p. 89.

89. Par exemple $\S 9$ de l'édition du texte.

90. $\S 1$ et $\S 11$ de l'édition du texte.

91. Ce couple fraternel rappelle également le duo, dont la relation est certes plus complexe, formé par Claire et François d'Assise.

92. Sir. 3,$18 ; \S 4$ de l'édition du texte. Il est dit au contraire de Salomon au Livre des Rois qu'il « surpassa tous les rois du monde en richesses et en sagesse "; cf. 1 R. 10, 23. 93. J. Le Goff, Saint Louis, op. cit., p. 395-396 ; Geoffroy de Beaulieu, Vita, dans Le procès de canonisation de saint Louis (1272-1297). Essai de reconstitution, op. cit., p. 33.

94. $§ 14$ de l'édition du texte.

95. A. Garreau, Bienheureuse Isabelle de France, soeur de saint Louis, Paris, 1955, p. 45.

96. J. Le Goff, Saint Louis, op. cit., p. 748.

97. J.-G. Bougerol, « Saint Bonaventure et le roi saint Louis », dans Saint Bonaventure, 1274-1974, II, Grottaferratta, 1973, p. 469-493.

98. Cité par J. Le Goff, Saint Louis, op. cit., p. 373.

99. Ibid., p. 750.

100. Geoffroy de Beaulieu, Vita, dans Le procès de canonisation de saint Louis (1272-1297).

Essai de reconstitution, op. cit., p. 33 ; J. Le Goff, Saint Louis, op. cit., p. 759.

101. $\S 12$ de l'édition du texte.

102. $\S 21$ de l'édition du texte.

103. Guillaume de Saint-Pathus, Vie de saint Louis, op. cit., p. 68. 
104. $\S 18$ de l'édition du texte ; cf. Guillaume de Saint-Pathus, Vie de saint Louis, op. cit., p. 94 .

105. § 22 de l'édition du texte.

106. J. Le Goff, Saint Louis, op. cit., p. 778.

107. § 24 de l'édition du texte.

108. Je remercie vivement Jacques Dalarun de m'avoir suggéré cette hypothèse. Selon Thomas de Pavie, les compagnes de Claire sont appelées « dames » car François d'Assise leur refusait cette appellation de « sœurs »; cf. J. Dalarun, François d'Assise : un passage. Femmes et féminité dans les écrits et les légendes franciscaines, trad. fr., Arles, 1997, p. 65-66. 109. § 12 de l'édition du texte.

110. $\S 14$ de l'édition du texte.

111. $\S 10$ de l'édition du texte.

112. C. Casagrande et $\mathrm{S}$. Vecchio, Les péchés de la langue. Discipline et éthique de la parole dans la culture médiévale, trad. fr., Paris, 1991, p. 14 et 114. A. Cabantous, Histoire du blasphème en Occident (XVI ${ }^{e}-X_{X}{ }^{e}$ siècles), Paris, 1998, p. 9-16.

113. J. Le Goff, « Saint Louis et la parole royale », dans J. Dufournet et L. Harf dir., Le prince et son historien. La Vie de saint Louis de Joinville, Paris, 1997.

114. Geoffroy de Beaulieu, Vita, dans Le procès de canonisation de saint Louis (1272-1297). Essai de reconstitution, op. cit., p. 33.

115. D. O'Connell, Les propos de saint Louis, Paris, 1974.

116. J. Le Goff, Saint Louis, op. cit., p. 604-605 ; J. Richard, Saint Louis, op. cit., p. 286-287.

117. $\S 25$ de l'édition du texte.

118. § 23 de l'édition du texte; cf. AN L 1020 ; G. Mlynarczyk, Ein

Franziskannerinnenkloster im 15. Jahrhundert, op. cit., p. 35.

119. § 18 de l'édition du texte.

120. J.-L. Flandrin, Un temps pour embrasser. Aux origines de la morale sexuelle occidentale ( $\mathrm{VI}^{e}-\mathrm{XI}^{e}$ siècles), Paris, 1983 ; J. Le Goff, Saint Louis, op. cit., p. 734-735.

121. Guillaume de Nangis, Gesta Ludovici IX, dans Recueil des historiens des Gaules et de la France, F. Daunou et J. Naudet éd., XX, Paris, 1840, p. 402 : « si la proximité de son épouse, en raison de la fragilité humaine, lui faisait ressentir des mouvements désordonnés de la chair, il se levait du lit et se promenait dans la chambre jusqu'à ce que la révolte de sa chair fût calmée. »

122. $§ 13$ de l'édition de texte.

123. Cf. P. Nagy, Le don des larmes : un instrument spirituel en quête d'institution ( $V^{e}-X I I I^{e}$ siècles), Paris, 2000.

124. Geoffroy de Beaulieu, Vita, dans Le procès de canonisation de saint Louis (1272-1297). Essai de reconstitution, op. cit., p. 42 ; J. Le Goff, Saint Louis, op. cit., p. 875-876.

125. Claire verse une telle abondance de larmes que les sœurs se mettent à pleurer également ; cf. Thomas de Celano, Sainte Claire d'Assise, sa vie et ses miracles, op. cit., p. 71. 126. $\S 43$ de l'édition du texte.

127. «Bonifacii VIII, sermones et bulla de canonisatione sancti Ludovici regis Francie », dans Recueil des historiens des Gaules et de la France, N. de Wailly, L. Delisle et C. Jourdain éd., XXIII, Paris, 1876, p. 150.

128. $\S 54$ de l'édition du texte.

129. $§ 55$ de l'édition du texte.

130. A. Vauchez, La sainteté en Occident aux derniers siècles du Moyen Âge, op. cit., p. 519.

131. Guillaume de Saint-Pathus, Vie de saint Louis, op. cit., p. 73. 
132. Ces statues sont réalisées vers 1220-1230. Cf. A. Erlande-Brandenburg, Les sculptures de Notre-Dame, Paris, 1986, p. 10-11.

133. Cf. Geoffroy de Beaulieu, Vita, dans Le procès de canonisation de saint Louis

(1272-1297). Essai de reconstitution, op. cit. ; Guillaume de Saint-Pathus, Vie de saint Louis, op. cit.

134. P. Arturi a Monasterio, Martyrologium franciscanum, $2^{\mathrm{e}}$ éd., Paris, 1753, p. 424 ;

J. Pien, G. Cuypers, J. Stiltingh, Acta sanctorum, Augusti, VI, op. cit., p. 796.

135. Paris, BnF, fr. 11662.

136. À propos de la tradition du texte et des différents manuscrits ayant existé, cf. mon introduction à l'édition du texte, infra, p. 76-78.

137. Le pape Jules II adresse aux religieuses une bulle dans laquelle il leur octroie l'autorisation de célébrer l'office divin pour Isabelle le 19 janvier 1517 ; cf. G. Duchesne, Histoire de l'abbaye royale de Longchamp, Paris, 1905, p. 8.

138. Paris, BnF, fr. 6214 : épitaphe d'Isabelle de France. J. Pien, G. Cuypers, J. Stiltingh, Acta sanctorum, Augusti, VI, op. cit., p. 792.

139. Paris, BnF, lat. $912, \mathrm{xvi}^{\mathrm{e}}$ siècle. L. Oliger, « Le plus ancien office liturgique de la bienheureuse Isabelle de France ", dans Miscellanea Giovanni Mercati, II : Letteratura medioevale, Vatican, 1946, p. 484-508.

140. Lourcine-lès-Saint-Marcel : abbaye de Clarisses fondée vers 1270 par Thibaud V comte de Champagne et Isabelle sa femme, fille de saint Louis, et transférée à Paris vers 1287, sur l'invitation de Marguerite de Provence. Dom L. H. Cottineau, Répertoire topobibliographique des abbayes et prieurés, Mâcon, 1937.

141. G. de Paris, Histoire de la fondation et de l'évolution de l'ordre des frères mineurs au XIII siècle, Rome, 1982, p. 609.

142. Abbaye de Clarisses fondée en 1248 par Thibaut IV, comte de Champagne, beaupère d'Isabelle de Navarre. Dom L. H. Cottineau, Répertoire topo-bibliographique des abbayes et prieurés, op. cit.

Abbaye de Clarisses fondée en 1254 dans le domaine d'Alphonse de Poitiers, comte de Toulouse depuis son mariage en 1251 avec Jeanne de Toulouse. Gallia Christiana, XIII, p. 146.

143. AN L $1027 n^{\circ} 5$, inventaire de l'abbaye de Longchamp (1325) : « La vie ma dame qui nous funda $»$.

144. AN L $1029 n^{\circ} 37$, préface de Pierre Perrier pour une inachevée Vie de sainte Isabelle de France, 1699 ; édition par S. L. FIELD, The Princess, the Abbess, and the Friars : Isabelle of France (1225-1270) and the Course of Thirteenth-Century Religious History, Ph. D. Diss., Northwestern University, 2002, p. 419-427.

145. J. Pien, G. Cuypers et J. Stiltingh, Acta Sanctorum, Augusti, VI, Anvers, 1743, p. 787.

146. Il n'existe pas de liste détaillée des livres de l'abbaye avant leur dispersion. Cf. AN S 4418, déclaration et actes administratifs de 1790 : «Dans la salle de la communauté : six corps des bibliothèques contenant mil quatre vingt trois volumes de livres de dévotion, et histoire, parmi lesquels livres de dévotion il y a au moins deux cents volumes vieux breviaires et autres de nulle utilité »; G. Duchesne, Histoire de l'abbaye royale de Longchamp (1255 à 1789), Paris, 1906, p. 109-114.

147. À ce sujet, on peut lire dans J. C. F. Hoefer dir., Nouvelle biographie générale, XXIII, Paris, 1861, col. 336-337, article « Harcourt (Agnès d') » : « Le manuscrit est conservé aux Archives impériales (c'est un rouleau de 8 feuillets de parchemin, cousus à la suite les uns des autres et de sept pieds de long) ». Cette affirmation, selon laquelle le manuscrit était aux Archives nationales en 1861 et aurait disparu par la suite, est reprise dans la thèse de G. Trouillard, Études sur la discipline et l'état intérieur des abbayes 
de l'ordre des Urbanistes et principalement de l'abbaye de Longchamp du XIII siècle au XVIII', thèse de l'École des Chartes, 1896. Nous remercions vivement les Archives départementales du Loir-et-Cher de nous avoir renseignés au sujet du manuscrit original de cette thèse, conservé sous la cote F 458-459. Guy Trouillard estimait que le manuscrit conservé aux Archives nationales avait dû en sortir entre 1861 et 1896. Cette affirmation doit nécessairement être une erreur. On a cru en 1861 que le manuscrit médiéval se trouvait aux Archives nationales sous la cote L 1029 n 37, alors qu'il s'agit d'une description de celui-ci dans le manuscrit de Pierre Perrier. Nous remercions Ghislain Brunel d'avoir pris le temps de nous aider à vérifier cette affirmation.

148. AN L $1027 \mathrm{n}^{\circ} 5$, inventaire de l'abbaye de Longchamp (1325) : «I autre livre ou est la rieule, est en françois et en latin, et la vie madame. ».

149. AN L $1028 \mathrm{n}^{\circ} 5$, inventaire de l'abbaye de Longchamp (31 mai 1448) : « Item, II livres de la ruylle en latin et en franscois, en l'un y a la vie madame sainte Claire, en l'aultre la Vie madame Isabel, notre sainte mere. "; AN L 1028 n 7 , inventaire (31 octobre 1467) : «Item, II livres de la rigle en latin et en francoys, en l'ung est la Vie madame sainte Claire, en l'aultre la Vie madame Ysabel qui nous fonda, notre sainte mere. »; AN L $1028 \mathrm{n}^{\circ}$ 9, inventaire (6 mars 1483) : « Item, deux livres de la reigle, en l'ung est la Vie sainte Claire, et en l'aultre la Vie sainte Elysabeth nostre sainte mere, qui nous funda, deux cayers de la Pacience Griseledis et ung cayer de la Vie saint Denis ». Cf. l'édition de ces inventaires dans G. MLYNARCZYK, Ein Franziskannerinnenkloster im 15. Jahrhundert. Edition und Analyse von Besitzinventaren aus der Abtei Longchamp, Bonn, 1987, p. 298 n $^{\circ} 266$, p. $315, n^{\circ} 192$, p. $334, n^{\circ} 207$. Le manuscrit de la Vie de sainte Claire en français est actuellement conservé aux Archives nationales, sous la cote LL 1601. Cf. F. Berriot, « Les manuscrits de l'abbaye de Longchamp aux Archives de France et la Vie de sainte Claire inédite (début xive) ", Archivum Franciscanum Historicum, 79, 1986, p. 329-358.

150. L. Delisle, Le cabinet des manuscrits de la Bibliothèque nationale, III, Paris, 1881, p. 158, $\mathrm{n}^{\circ} 968$.

151. L. Douët d'Arcq éd., Inventaire de la bibliothèque du roi Charles VI fait au Louvre en 1423, Paris, 1867, p. 99, $\mathrm{n}^{\circ} 356$ [340].

152. BnF, fr. 13747 , f. $127-141 v^{\circ}$. Cette copie est en grande partie de la main d'Antoine Le Maistre, d'après le manuscrit conservé à Longchamp sous forme de rouleau, et achevée le 5 janvier 1653. Édition par S. L. FIELD, The Princess, the Abbess, and the Friars, op. cit., p. 367-393.

153. S. L. FieLD, The Princess, the Abbess, and the Friars, op. cit., p. 16-17.

154. C. Du Fresne, sieur Du Cange, Histoire de saint Louys IX du nom roy de France écrite par Jean sire de Joinville sénéchal de Champagne, Paris, 1668, p. 169-181. Cet ouvrage est conservé à la Réserve de la BnF. Sur Du Cange, voir L. Feugère, Étude sur la vie et les ouvrages de Du Cange, Paris, 1852.

155. Cf. introduction du texte infra. Érudit et auditeur des comptes de Paris. Cf.

A. Bruel, « Notes de Vyon d'Hérouval sur les captisés et les convers et sur les enquêteurs royaux au temps de saint Louis et de ses successeurs ", Bibliothèque de l'École des Chartes, 28, 1867, p. 610-621.

156. Ces érudits semblent avoir eu le souci de restituer fidèlement le contenu du texte. Cf. S. L. FIELD, The Princess, the Abbess, and the Friars, op. cit., p. 24-25.

157. $\S 54$ et 55 . La copie de Le Maistre porte pourtant comme celle de Du Cange la mention « XL Miracles ». 
158. J. Pien, G. Cuypers et J. Stiltingh, Acta Sanctorum, Augusti, VI, Anvers, 1743, p. 798-808.

159. École nationale des Chartes, Conseils pour l'édition des textes médiévaux, I, Paris, 2001.

160. Isabelle de France, unique sœur de Louis IX, est née en mars 1225.

161. « Monseigneur » LM (Antoine Le Maistre édité par Sean Linscott Field). Le terme « monsieur » doit être une erreur de transcription de l'époque moderne.

162. Charles d'Anjou est né en mars 1227. C'est le dernier fils de Louis VIII et Blanche de Castille. En 1266, il est reconnu roi de Sicile par le pape. Après les Vêpres Siciliennes de 1282, il conserve le royaume de Naples, siège d'une cour brillante. Il meurt à Foggia en 1285. Le terme " germain " signifie « né de même père et mère ".

163. Personnes.

164. Son genre de vie.

165. « Du » LM.

166. Louis VIII est le père d'Isabelle.

167. Philippe Auguste.

168. Blanche de Castille est née en 1188. Elle épouse en 1200 le futur Louis VIII. Régente de 1226 à la majorité de Louis IX en 1234, elle conserve une grande influence jusqu'à sa mort en 1252.

169. Alphonse VIII, roi de Castille, vécut de 1155 à 1214. L'expression « roi d'Espagne » est une modification de l'époque moderne ; cf. n. 14 de notre article « Isabelle de France, sœur de saint Louis : la vierge savante. Étude de la Vie d'Isabelle de France écrite par Agnès d'Harcourt » supra, p. 57. La version de Le Maistre l'utilise également : au roy d'Espaigne $L M$.

170. Louis VIII et Blanche de Castille ont eu trois enfants morts en bas âge, dont peutêtre une autre fille ; cf. J. Le Goff, Saint Louis, Paris, 1996, p. 956.

171. «Il» om. LM.

172. "S'entention » $L M$.

173. Cf. n. 18 de notre article.

174. Matt. 11, 29.

175. « De France » om., " quar » add. LM.

176. « Ce » add. LM.

177. « Et » add. LM.

178. Sir. $3,18$.

179. « Et cette » LM.

180. Jeux, divertissements.

181. « Sa mere » add. LM.

182. L'épitaphe d'Isabelle de France nous renseigne sur ses vêtements lorsqu'elle vit à proximité de Longchamp. Cf. Paris, BnF, fr. 6214, f. 2 . Le texte, daté du Xv ${ }^{\mathrm{e}}$ siècle selon les Acta Sanctorum, précise : « Elle estoit moult humble en vesture et estoient ses robes de gros drap de burel et les vestoit jusques a tant qu'elles fussent usees et percees. »

183. " Juree » LM.

184. Ses parents.

185. Il s'agit du fils de Frédéric II, Conrad de Hohenstaufen, le futur Conrad IV.

186. " Onques...ne » : jamais.

187. Sinibaldo Fieschi (1195-1254), devenu le pape Innocent IV en 1243, a lutté notamment contre Conrad IV et Manfred.

188. « À » $L M$. 
189. « $\mathrm{Ce} »$ add. LM.

190. « Li » LM.

191. «Quan» $L M$.

192. « $\mathrm{A} » L M$.

193. Cf. BnF, fr. 13747 , f. 119 : lettres du pape Innocent IV du 22 juillet 1253 ; édition par S. L. Field, The Princess, the Abbess, and the Friars, op. cit., p. 399-400. AN L 250, nº 78 [olim $n^{\circ}$ 91] : 12 juin 1256. Bulle d'Alexandre IV, provenant des archives de Longchamp, approuvant le dessein d'Isabelle de vivre en perpétuelle virginité. B. Barbiche, Les actes pontificaux originaux des Archives nationales de Paris, I, Cité du Vatican, 1975, p. 328-329, $\mathrm{n}^{\circ} 855$.

194. Tout à fait, pleinement.

195. Il doit manquer un terme : la proposition « pour neant fust ce » signifie « bien qu'il ne fut pas »; cf. A. Tobler et E. Lommatzsch, Altfranzösisches Wörterbuch, Wiesbaden, 1963.

196. «Ses » LM.

197. « Ce » LM.

198. Ne pas accorder d'importance.

199. Marguerite de Provence, épouse de Louis IX, qui vécut de 1221 à 1295.

200. « Engregier, engrejer » : empirer, s'aggraver.

201. «Et envoia l'en » $L M$.

202. Louis IX est le frère aîné d'Isabelle, né en 1214. Il devient roi en 1226. Il meurt en 1270.

203. Chagrin, douleur.

204. « À Nanterre » $L M$.

205. Cette personne pourrait être une religieuse auteur du Miroir de l'âme écrit pour Blanche de Castille ; cf. G. Sivéry, Blanche de Castille, Paris, 1990, p. 226.

206. " Ataineux ", " ateineux » : acharné, violemment désireux. Cf. F. Godefroy, Dictionnaire de l'ancienne langue française et de tous ses dialectes $d u I^{e}$ au $X V^{e}$ siècle, Paris, 1881-1902.

207. « Pour aider sa fille » $L M$.

208. Clairement.

209. « Pour» add. LM.

210. «Fors que »: si ce n'est que, excepté.

211. Visage, face.

212. Appuyée, accoudée.

213. « Qu'il» $L M$.

214. Voyager.

215. « Devoit » LM.

216. Emballer, empaqueter.

217. En ce lieu-là.

218. « Monseigneur le sainct roy Loïz » LM.

219. Mahaut de Godarville fait partie des premières nonnes du monastère de Longchamp qui ont revêtu l'habit en 1260. Cf. Paris, BnF, fr. 11662 , f. $44 \mathrm{v}^{\circ}$ : liste des sœurs de l'abbaye de Longchamp.

220. « Tans » LM.

221. « Donrroit» $L M$.

222. Toutes sortes d'aliments, de nourriture.

223. Potage à base de poireaux ou d'autres légumes. 
224. «Baiens » LM. Crevé, en parlant de pois ou de fèves.

225. "Mout deligamment et l'escoutoit tout " add. LM.

226. Chapelle.

227. « Leus » LM.

228. Bien qu'elle ne soit pas une dame de religion. Cf. n. 53.

229. " Et de » LM.

230. Om. LM.

231. « Denoit» $L M$.

232. "Fracon » ou « fraillon » : petit houx.

233. « Ne » LM.

234. « $Y$ ces » LM.

235. «En l'Évangile» add. LM.

236. "Pouvres» LM.

237. Se tourmenter.

238. Commune de l'Oise, canton de Beauvais.

239. Il pourrait s'agir de la maladrerie de Saint-Cloud ; cf. AN L 1020, $n^{\circ} 19$ : 25 octobre 1264. Acquisition par les dames religieuses de Longchamp aux administrateurs de la maladrerie de Saint-Cloud de huit arpents de terre situés entre Neuilly et l'abbaye.

240. Flétrie ; cf. A. TOBLER et E. LOMMATZsCH, Altfranzösisches Wörterbuch, op. cit.

241. « Li » $L M$.

242. L'attitude d'Isabelle vis-à-vis de cette lépreuse rappelle celle de saint Louis avec le lépreux de Royaumont ; cf. J. Le Goff, Saint Louis, op. cit., p. 881.

243. Eût-elle été sa fille qu'elle n'en eût pas fait davantage ; cf. n. 53 .

244. Claire d'Assise, malade et contrainte de garder le lit, voulut donner l'exemple à ses sœurs en travaillant de ses mains pour faire des corporaux à offrir à toutes les églises de la plaine autour d'Assise ; cf. Thomas de Celano, Sainte Claire d'Assise, sa vie et ses miracles, M. Havard de la Montagne trad., Paris, 1917, p. 111.

245. Om. LM.

246. Il s'agit de parentes de Jean de Montfort, compagnon de saint Louis lors de sa première croisade. " Peironnelle » est sans doute sa sœur Pernelle de Montfort, qui fut abbesse de Port-Royal des Champs près de Paris et mourut après 1275 . Cf. M. J.

L'Hermitte, Précis sur la ville de Montfort-l'Amaury, et histoire chronologique des seigneurs de cette ville, Paris, 1825 , p. 89.

247. L'abbaye de Saint-Antoine-des-Champs fut fondée en 1198 par Foulques, curé de Neuilly-sur-Marne. L'église abbatiale fut fondée entre 1220 et 1230 par Louis IX. La dédicace fut faite le 2 juin 1233 par Guillaume évêque de Paris, en présence du roi et de Blanche de Castille. Cf. H. Bonnardot, L'abbaye royale de Saint Antoine des Champs de l'ordre de Citeaux, Paris, 1881.

248. " Monseigneur» LM. Cf. § 1 et en note.

249. "Qui mourut en Montpencier » add. LM.

250. AN J $403 \mathrm{n}^{\text {os }} 2$ et $2^{\text {bis }}$ : testament de Louis VIII, juin 1225. A. Teulet éd., Layettes du trésor des chartes, II, Paris, 1866, p. 54-55, nº 1710 : «Item donamus et legamus Elisabet karissima filie nostre viginti milia librarum. ".

251. "Assener » : désigner. Cf. F. Godefroy, Dictionnaire de l'ancienne langue française et de tous ses dialectes $d u I^{e}$ au XV siècle, op. cit., I.

252. Jeudi saint.

253. "Ce qu'ele en deust fere » LM. 
254. Il s'agit du chancelier de Paris Aymeric de Veire. En 1232, il était maître de théologie et archidiacre de Paris. En 1248, il devient chanoine de Paris et régent en théologie. En 1250, il succède à Gauthier de Château-Thierry dans la charge de chancelier. Il meurt vers 1262-1263. Cf. P. Glorieux, Répertoire des maîtres en théologie de Paris au XIII ${ }^{e}$ siècle, I, Paris, 1933, p. 332.

255. « À » add. LM.

256. « Lui » serait plus vraisemblable.

257. Il est né en 1221 près de Viterbe, étudie la théologie aux Cordeliers de Paris, disciple d\&\#39

\section{RÉSUMÉS}

Isabelle de France est la sœur de Louis IX et la fondatrice du monastère des Clarisses de Longchamp. Dans le contexte de la canonisation en cours du roi Louis IX, Charles d'Anjou commande à Agnès d'Harcourt, alors abbesse de Longchamp, une Vie d'Isabelle de France. Le texte est rédigé en prose et en langue vernaculaire vraisemblablement entre 1279 et 1281 . La vie et les miracles d'Isabelle constituent un nouvel argumentaire en faveur de la sainteté dynastique capétienne. La princesse est présentée comme une vierge savante; sa vie emprunte à la fois à un modèle féminin de sainteté proche de Claire d'Assise, et à un modèle royal et masculin, celui de saint Louis. Une édition de ce texte est proposée à la suite du commentaire.

Isabelle de France, Saint Louis' Sister: the Literate Virgin. A Study of the Vie d'Isabelle de France written by Agnes d'Harcourt. Isabelle of France was king Louis IX of France's sister ; she established an abbey of Clarisses in Longchamp. When the king's canonization was in process, Charles d'Anjou asked Agnes d'Harcourt, abbess of Longchamp, for a Vie d'Isabelle de France. This text was written in prose and in vernacular language between 1279 and 1281. Isabelle's life and miracles provide new elements to support the idea of the whole capetian dynasty's sanctity. The princess appears as a literate virgin; her life refers both to a model of feminine sanctity inspired of Claire $\mathrm{d}$ 'Assise, and to a masculine and royal model, based on the hagiography of saint Louis. An edition of the text follows this study.

\section{INDEX}

Mots-clés : Isabelle de France, sainteté féminine, dynastie capétienne

Keywords : Isabelle of France, feminine sanctity, capetian dynasty, Louis IX, clarisses, Longchamp

\section{AUTEUR}

\section{ANNE-HÉLÈNE ALLIROT}

Centre de recherche d'Histoire Sociale et Culturelle de l'Occident, Université de Paris-X Nanterre, 200, avenue de la République, F-92000 Nanterre 